\title{
FERMIONIC CHERN-SIMONS FIELD THEORY FOR THE FRACTIONAL HALL EFFECT
}

\author{
ANA LOPEZ \\ Department of Physics, Theoretical Physics, Oxford University, \\ 1 Keble Rd., Oxford OX1 3NP, United Kingdom \\ EDUARDO FRADKIN \\ Department of Physics, University of Illinois at Urbana-Champaign, \\ 1110 W. Green St., Urbana, Ill 61801, USA
}

\begin{abstract}
We review the fermionic Chern-Simons field theory for the Fractional Quantum Hall Effect (FQHE). We show that in this field theoretic approach to the problem of interacting electrons moving in a plane in the presence of an external magnetic field, the FQHE states appear naturally as the semiclassical states of the theory. In this framework, the FQHE states are the unique ground states of a system of electrons on a fixed geometry. The excitation spectrum is fully gapped and these states can be viewed as infrared stable fixed points of the system. It is shown that the long distance, low energy properties of the system are described exactly by this theory. It is further shown that, in this limit, the actual ground state wave function has the Laughlin form. We also discuss the application of this theory to the problem of the FQHE in bilayers and in unpolarized single layer systems.
\end{abstract}

\section{Introduction}

The fermionic Chern-Simons field theory for the FQHEQ was motivated by Jain's observation that the Laughlin wave function could be reinterpreted as the wave function of charge-flux composites filling up a lowest Landau level, (i. e., a Vandermonde determinant), with each unit of charge being bound to an even number of flux quanta. Following Jain we will refer to these charge-flux composite particles, as composite fermions 2.3 In Jain's picture, the electrons "nucleate" flux to screen enough of the external magnetic field, so that the "composite fermions" exactly fill an integer number of Landau levels associated with the unscreened part of the field. In this formulation, the FQHE is an Integer Quantum Hall Effect (IQHE) of the bound states.

The starting point of Jain's approach was the Laughlin wave function. In 1989, he proposed to write it in the suggestive factorized form

$$
\Psi_{\frac{1}{m}}\left(z_{1}, \ldots, z_{N}\right)=\prod_{i<j}\left(z_{i}-z_{j}\right)^{m-1} \chi_{1}\left(z_{1}, \ldots, z_{N}\right)
$$


where $\chi_{1}$ is the wave function for a completely filled lowest Landau level

$$
\chi_{1}\left(z_{1}, \ldots, z_{N}\right)=\prod_{i<j}\left(z_{i}-z_{j}\right) \exp \left(-\sum_{i=1}^{N} \frac{\left|z_{i}\right|^{2}}{4 \ell^{2}}\right) .
$$

The phases associated with the first factor in Eq. 1 1 represent an even number $(m-1)$ of fluxes that are attached to each coordinate $z_{i}$ where an electron is present. It is a crucial feature of this picture that the electrons bind to an even number of flux quanta and, in this way, they retain their fermion character. This observation gave rise to the picture of the FQHE as a ground state of "composite fermions", where a composite fermion is an electron bound to an even number of fluxes. In this formulation, the main role of the long range correlations is to make it possible for the electrons to "nucleate" flux, and the FQHF can be interpreted as an Integer Quantum Hall Effect of the bound states 년.

The question that naturally arises here is how does a system of electrons in an external magnetic field manage to turn the correlations, which result from the electron-electron interactions, into "nucleated fluxes". In this Chapter we show that the key to the answer is the Chern-Simons gauge theory. In 1982 Wilczek's observed 4 that a particle current coupled to a Chern-Simons (CS) gauge field produced states with fractional statistics through the binding of particles to fluxes. Thus, if we are to get the Laughlin wave function by attaching $(m-1)$ fluxes to each electron, as suggested by Jain, it is natural to guess that the "right" theory must contain fermions (electrons) coupled to Chern-Simons gauge fields with an appropriate value of the Chern-Simons coupling constant $\theta$. What is less clear is the origin of such a Chern-Simons gauge field for the problem of interacting electrons in a magnetic field. In Quantum Field Theory, where the CS gauge theory was first introduced the Chern-Simons term in the action originates from the Parity Anomaly of relativistic fermions in $2+1$ dimensions. Clearly, the electrons which live in quasi two-dimensional electron gases, are not relativistic. Also the real three dimensional system one is dealing with, neither breaks Parity by itself nor as a result of the presence of the device. The Chern-Simons gauge field must then be a result of the dynamics of interacting two dimensional electrons in the presence of an external magnetic field. For our purposes, the most important feature of the Chern-Simons term is not its relativistic invariance, but the fact that it is the only local gauge invariant theory which yields bound states of particles and fluxes.

In this Chapter we derive a field theory for the FQHE in the fermion language in which the Chern-Simons gauge field appears explicitly in the problem 
of interacting electrons in a magnetic field. We do so by first considering a theory in which the electrons, in addition to their mutual interaction, are coupled to both an external electromagnetic field and a Chern-Simons gauge field. In doing so, we use the fact that if the coefficient of the Chern-Simons term is chosen in such a way that an even number of flux quanta get attached to each electron, all the physical amplitudes calculated in this theory are identical to the amplitudes calculated in the standard theory, in which the CS field is absent (see referencet). We further show that the Laughlin state is the semiclassical approximation of this theory. In the "classical" (i.e., mean-field) approximation we get a picture very close to the one proposed by Jain. This theory, not only explains where do the fluxes come from, but also allows for the systematic calculation of corrections around this state in a manner similar to the semiclassical approximation in quantum mechanics and to the Random Phase Approximation (RPA) in many-body theory. A conceptually important feature of the Fermionic Chern-Simons theory is that its microscopic fields are composite fermions. Hence, at the level of mean field theory, it describes an Integer Quantum Hall (IQH) state of composite fermions. Nevertheless, quantum fluctuations about this mean field state change this picture in a qualitatively fundamental way. They not only give the correct FQHE but also change the charge and statistics of the quasiparticles which become fractionally charged anyons, in agreement with the predictions of Laughlin's theory. The reason behind this behavior is the fact that the Chern-Simons fields require a local relation between charge and flux. This relation is replaced at the level of mean field theory by an average. The fluctuations correct this effect, forcing charge fluctuations to be accompanied by the necessary flux fluctuations. At long wavelengths, the leading gaussian fluctuations enforce this flux-charge relation exactly, leading to the exact values of the Hall conductance, charge and statistics of the excitations as well as to the exact saturation of the sum rules. However, at shorter distances and higher energies, it is necessary to go beyond gaussian fluctuations to account for the correct correlations.

This Chapter is organized as follows. In Section 2 we derive the Fermionic Chern-Simons theory of the FQHE for a single layer fully polarized two dimensional electron gas (2DEG). In Section 3 we discuss the electromagnetic response functions and the spectrum of collective modes. In Section 1 we generalize this theory to the problem of the FQHE in bilayers and partially polarized and spin singlet FQH states in single layers. In Section 5 we use the Fermion Chern-Simons theory to derive the asymptotic long distance form of the wave functions of the FQH states. Finally, in Section 6 we present a summary of our results. 


\section{The Fermionic Chern-Simons Theory for the Fractional Quantum Hall Effect}

Consider a system of $N$ electrons moving on a plane in the presence of an external uniform magnetic field $B$ perpendicular to the plane. The electrons will be assumed to have an interparticle interaction governed by a pair potential $V(|\vec{r}|)$, for two electrons separated a distance $|\vec{r}|$ on the plane. In this section we will assume that the magnetic field is sufficiently large so that the system is completely polarized and thus we will can ignore the spin degrees of freedom. In Section 1 we will consider polarization effects. The eigenstates $\Psi\left(\vec{x}_{1}, \ldots, \vec{x}_{N}\right)$ are eigenfunctions of the (first quantized) Hamiltonian $\hat{H}$

$$
\hat{H}=\sum_{i=1}^{N}\left\{\frac{1}{2 M}\left(\vec{p}_{j}-\frac{e}{c} \vec{A}_{j}\left(\vec{x}_{j}\right)\right)^{2}+e A_{0}\left(\vec{x}_{j}\right)\right\}+\sum_{i<j} V\left(\left|\vec{x}_{i}-\vec{x}_{j}\right|\right)
$$

where we have included the coupling to both the electromagnetic vector potential $\vec{A}$ and scalar potential $A_{0}$. Hence, we are dealing with $N$ spinless fermions of charge $-e$ and mass $M$.

Our goal is to show that this system is equivalent to the same system but coupled to an additional statistical vector potential $a_{\mu}(\mu=0,1,2)$ whose dynamics is determined by the Chern-Simons action, $S_{\mathrm{CS}}$

$$
S_{\mathrm{CS}}=\int d^{3} x \frac{\theta}{4} \epsilon_{\mu \nu \lambda} a^{\mu} \mathcal{F}^{\nu \lambda} \text {. }
$$

for a suitably chosen value of $\theta$. In Eq. 国 $x_{0}, x_{1}$ and $x_{2}$ represent the time and the space coordinates of the electrons respectively, and $\mathcal{F}^{\nu \lambda}$ is the field tensor for the statistical gauge field

$$
\mathcal{F}^{\nu \lambda}=\partial^{\nu} a^{\lambda}-\partial^{\lambda} a^{\nu}
$$

The equivalent theory has a Hamiltonian $\hat{H}^{\prime}$ which is identical to $\hat{H}$, given in Eq. 3, except for the fact that the electrons are also coupled to the statistical vector potential $a_{\mu}$. This is accomplished simply by setting

$$
\frac{e}{c} \vec{A} \rightarrow \frac{e}{c} \vec{A}+\vec{a} ; \quad e A_{0} \rightarrow e A_{0}+a_{0} .
$$

The Chern-Simons gauge theory is a topological field theory. This means that the expectation values of the observables in this theory do not depend on the distance ( $i . e$, the metric) between the points of space-time on which they act, but only on topological properties. Being a gauge theory, its observables are path ordered exponentials of the circulation of gauge fields on closed loops 
(Wilson loops) an their expectation values are functions of the linking numbers of these loops 7 . Since it is a topological theory, the action of the Chern-Simons gauge fields alone does not involve any energy scales. In fact, it is easy to see that the Hamiltonian is actually equal to zero (in the abscence of coupling to matter). We will now follow standard arguments and show that the content of the Chern-Simons action reduces to a constraint and to a set of commutation relations for the gauge fields. Indeed, let us expand the Chern-Simons action, coupled to an external matter current $j_{\mu}(x)$, in components to read

$$
S_{\mathrm{CS}}=\int d^{3} x\left\{\left[-j_{0}(x)+\theta \mathcal{B}(x)\right] a_{0}(x)+\theta a_{2}(x) \partial_{0} a_{1}(x)+\vec{a}(x) \cdot \vec{j}(x)\right\}
$$

where $j_{o}(\vec{x})$ is the particle density, $\vec{j}(\vec{x})$ is the matter current and $\mathcal{B}$ is the statistical flux. By direct inspection of Eq. 7, we see that the time component of the field $a_{0}(x)$ acts like a Lagrange multiplier field which imposes the constraint

$$
j_{0}(\vec{x})=\theta \mathcal{B}(\vec{x}) .
$$

which is the analog of Gauss' law for this theory. At the quantum level, Eq. 8 is an operator constraint which selects the physical space of states $i$. e. , the gauge invariant states are made of charge-flux composites. The second term of the Chern-Simons action Eq. I I implies that the momentum canonically conjugate to the field $a_{1}$ is just $\theta a_{2}$ and that, quantum mechanically they have to obey the equal-time commutation relations

$$
\left[a_{1}(\vec{x}), a_{2}\left(\vec{x}^{\prime}\right)\right]=\frac{i}{\theta} \delta^{(2)}\left(\vec{x}-\vec{x}^{\prime}\right) .
$$

Finally, the last term in the action of Eq. 7, says that the Hamiltonian is just

$$
H=-\int d^{2} x \vec{j}(\vec{x}) \cdot \vec{a}(\vec{x})
$$

which vanishes in the abscence of matter currents.

Thus, for arbitrary values of the Chern-Simons coupling constant $\theta$, the physical states are charge-flux composites: every particle of charge 1 carries a gauge flux equal to $1 / \theta$. Intuitively we expect that the wave functions for these composite particles should exhibit an Aharonov-Bohm effect which can be regarded as a change of statistics 8 . In Chern-Simons theory this statistical transmutation is seen in the following way. Imagine that we have $N$ particles which are sufficiently heavy so that their Feynman path integrals are dominated by reasonably smooth, well separated paths in $2+1$-dimensional space-time. Consider for simplicity the case of two particles and let $\Gamma_{1}$ and $\Gamma_{2}$ be their 
respective paths. Let us denote by $\Gamma=\Gamma_{1} \bigcup \Gamma_{2}$ their joint paths. Let us further define a current $j_{\mu}(x)$ which carries one unit of charge and with support on $\Gamma$. A typical amplitude for this 2-particle system is given by the Wilson loop expectation value 10

$$
\left\langle e^{i \oint_{\Gamma} d x_{\mu} a^{\mu}(x)}\right\rangle_{\mathrm{CS}}=\left\langle e^{i \int d^{3} x j_{\mu}(x) a^{\mu}(x)}\right\rangle_{\mathrm{CS}}=e^{\frac{i}{2 \theta} \nu_{L}[\Gamma]}
$$

where the expectation values are calculated in the Chern-Simons theory and $\nu_{L}[\Gamma]$ is the linking number of the path $\Gamma$. From here it follows that processes involving the exchange of one pair of particles differ by the phase factor $\exp \left(i \delta \Delta \nu_{L}[\Gamma]\right)$, where we have defined the statistical angle $\delta=\frac{1}{2 \theta}$ and $\Delta \nu_{L}[\Gamma]$ is the change of linking number. For an exchange process, $\Delta \nu_{L}[\Gamma]= \pm 1$ and the phase factor is just $\exp ( \pm i \delta)$. Thus, $\delta$ is regarded as the change (or shift) of statistics. By generalizing these arguments for an $N$-particle system we conclude that a system of fermions coupled to a Chern-Simons gauge field with coupling constant $\theta$ behaves like a system of anyons with statistical angle $\delta=\frac{1}{2 \theta}$, measured with respect to Fermi statistics 11 . If $\theta=\frac{1}{2 \pi} \frac{1}{2 s}$, where $s$ is an arbitrary integer, then $\delta=2 \pi s$ and the system still represents fermions.

In referencel we presented a detailed proof of the physical equivalence of two theories of particles coupled to a Chern-Simons gauge field with coupling constants $\theta$ and $\theta l$ such that

$$
\frac{1}{\theta^{\prime}}=\frac{1}{\theta}+2 \pi \times 2 s
$$

where $s$ is an arbitrary integer. There, we showed that the physical observables have exactly the same matrix elements in both theories and, consequently, that the theories are physically equivalent. In particular, a theory of interacting fermions is always equivalent to a family of theories of interacting fermions coupled to a Chern-Simons gauge field with coupling constant $\theta$ such that $\frac{1}{\theta}=2 \pi \times 2 s$. This result is the starting point of our analysis of the FQHE.

From Eq 11, it is apparent that the physics of these theories is a periodic function of the statistical angle, $\delta \rightarrow \delta+2 \pi \times$ integer. Even though these theories are exactly periodic, periodicity is broken in approximate, semiclassical approximations, such as in the Average Field Approximation that we will use here since one particular period will have to be chosen. This is an important issue since, at the perturbative level, the fluctuations around this mean-field are not able to restore periodicity as an exact property. The restoration of periodicity is a non-perturbative effect. In particular, operators which create excitations that change the amount of flux per particle in even multiples of the flux quantum are soliton operators which restore the periodicity broken by the mean-field. However, we can take advantage of the periodicity of the 
CS description to choose the period in which the mean-field-theory is simplest. This is the approach we take in this work to attack the FQHE. As it will be apparent in the next Section, at the mean-field level, this approach reproduces Jain's construction of the FQHE states. Recently, Kivelson, Lee and Zhang 12 have forcefully argued that periodicity plays a central role in the determination of the global phase diagram of the 2DEG in a strong magnetic field.

In second quantized language, what we have proven is the physical equivalence of theories whose dynamics are governed by the actions (in units in which $\hbar=1) \mathcal{S}_{\theta}$ and $\mathcal{S}_{\theta^{\prime}}$ which are defined by

$$
\begin{aligned}
\mathcal{S}_{\theta} & =\int d^{3} z\left\{\psi^{*}(z)\left[i D_{0}+\mu\right] \psi(z)-\frac{1}{2 M}|\vec{D} \psi(z)|^{2}+\frac{\theta}{4} \epsilon_{\mu \nu \lambda} a^{\mu} \mathcal{F}^{\nu \lambda}\right\} \\
& -\frac{1}{2} \int d^{3} z \int d^{3} z^{\prime}\left(|\psi(z)|^{2}-\bar{\rho}\right) V\left(\left|\vec{z}-\vec{z}^{\prime}\right|\right)\left(\left|\psi\left(z^{\prime}\right)\right|^{2}-\bar{\rho}\right) .
\end{aligned}
$$

where $\bar{\rho}$ is the average particle density, provided that $\theta$ and $\theta^{\prime}$ satisfy Eq. 12. In Eq. $13 \psi(z)$ is a second quantized Fermi field, $\mu$ is the chemical potential and $D_{\mu}$ is the covariant derivative which couples the fermions to both the external electromagnetic field $A_{\mu}$ and to the statistical gauge field $a_{\mu}$

$$
D_{\mu}=\partial_{\mu}+i \frac{e}{c} A_{\mu}+i a_{\mu} .
$$

In particular, a theory of interacting fermions ( which has $s=0$ ) is equivalent to a family of theories of fermions with $\frac{1}{\theta}=2 \pi \times$ even integer.

\subsection{The Semiclassical Limit and The Laughlin Ground State}

In the remaining of this Section we will show that the semiclassical limit of the theory described by the action $S_{\theta}$ of Eq. 13 , with $\frac{1}{\theta}=2 \pi(m-1)$, yields the same physics as the Laughlin state. In order to prove this statement we will develop a semiclassical approach to this problem. As a by-product, our formalism provides for a systematic procedure to compute corrections to the Laughlin approximation. This is, to the best of our knowledge, the first formalism for which the Laughlin ansatz arises as the first of a series of approximations.

Consider the quantum partition function for this problem ( at $T=0$ )

$$
\mathcal{Z}=\int \mathcal{D} \psi^{*} \mathcal{D} \psi \mathcal{D} a_{\mu} \exp \left(i S_{\theta}\right) .
$$

We will treat this path-integral in the semiclassical approximation. In order to do that, we will first integrate-out the fermions and treat the resulting bosonic 
theory within the Saddle Point Expansion (SPE) characteristic of semiclassical approaches to quantum mechanics and quantum field theory 13, 14. In terms of Feynman diagrams, the semiclassical expansion is an expansion in powers of the number of fermion loops (bubbles). Thus, the leading semiclassical approximation (gaussian fluctuations) is equivalent to the conventional RPA of many-body physics. It is easy to see that the formal expansion parameter that organizes the SPE is the filling fraction $\nu$. This is not surprising since RPA is a high density approximation 15 . However, for the FQHE, the filling fraction $\nu$ is never large but typically of order one. We will show below that, in spite of this, the SPE will still be very useful since, for special values of the filling fraction, it will enable us to construct a set of ground states for which the spectrum is fully gapped. Thus, for those states the SPE yields a perturbative expansion free of infrared divergencies. However we will also encounter a set of fractions for which there is no gap and the SPE (or equivalently, RPA) is infrared divergent at every order. Interestingly enough, the set of fractions for which the SPE predicts a fully gapped state coincides with the main fractions of the FQHE. These are the states in Jain's hierarchy.

In the absence of electron-electron interactions, the fermions can be integrated out immediately since the action becomes quadratic in Fermi fields. In the presence of interactions this is no longer possible since the interaction term in the action Eq. 13 spoils this feature. However, the physical states used in deriving the path integral must obey the local charge-flux constraint of the Chern-Simons theory. Hence, it is legitimate to replace the charge density $j_{0}(x)$ by $\theta \mathcal{B}(x)$ in the pair-interaction term of the action, at all points of space-time $x$ and to write the pair-interaction term of Eq. 13 in the form

$$
\left.\mathcal{S}_{\mathrm{int}}=\frac{1}{2} \int d^{3} z \int d^{3} z^{\prime}(\theta \mathcal{B}(z)-\bar{\rho}) V\left(z-z^{\prime}\right)\left(\theta \mathcal{B}\left(z^{\prime}\right)-\bar{\rho}\right)\right)
$$

where $V\left(z-z^{\prime}\right)$ represents the instantaneous pair interaction $i . e$.,

$$
V\left(z-z^{\prime}\right)=V\left(\left|\vec{z}-\vec{z}^{\prime}\right|\right) \delta\left(t-t^{\prime}\right) .
$$

We will assume that the pair potential has either the Coulomb form, i. e., $V(|\vec{r}|)=\frac{q^{2}}{r}$, or that in momentum space it satisfies that $\tilde{V}(\vec{Q}) \vec{Q}^{2}$ vanishes at zero momentum. This includes the case of ultralocal potentials, $(i$. e., with a range smaller or of the same order as the cyclotron length $\ell$ ), in which case we can set $\tilde{V}=0$, or short range potentials with a range longer that $\ell$ such as a Yukawa interaction.

Note that since we are dealing with a gauge theory, a gauge has to be specified in order to make the functional integral well defined. We will assume 
from now on that a gauge fixing condition has been imposed but, for the moment, we will not make any specific choice of gauge.

The partition function $\mathcal{Z}$ can be written in the form of a functional integral involving the Fermi fields $\psi$, and the statistical gauge fields $a_{\mu}$. The Fermi fields can be integrated out without any difficulty yielding, as usual, a fermion determinant. The resulting partition function can thus be written in terms of an effective action $S_{\text {eff }}$ given by

$$
\begin{aligned}
S_{\mathrm{eff}} & =-i \operatorname{tr} \log \left[i D_{0}+\mu+\frac{1}{2 m} \vec{D}^{2}\right]+S_{\mathrm{CS}}\left(a_{\mu}-\tilde{A}_{\mu}\right) \\
& -\frac{1}{2} \int d^{3} z \int d^{3} z^{\prime}[\theta(\mathcal{B}(z)-\tilde{B}(z))-\bar{\rho}] V\left(z-z^{\prime}\right)\left[\theta\left(\mathcal{B}\left(z^{\prime}\right)-\tilde{B}\left(z^{\prime}\right)\right)-\bar{\rho}\right]
\end{aligned}
$$

where $D_{0}$ and $\vec{D}$ are the covariant derivatives of Eq. 14 and $S_{\mathrm{CS}}$ is the ChernSimons action Eq. 4 . The field $\tilde{A}_{\mu}$ represents a small fluctuating electromagnetic field, with vanishing average everywhere, which will be used to probe the system. The electromagnetic currents will be calculated as first derivatives of $\mathcal{Z}$ with respect to $\tilde{A}_{\mu}$. The full electromagnetic response will be obtained in this way. Notice that we have used the invariance of the measure $\mathcal{D} a_{\mu}$ with respect to shifts, to move $\tilde{A}_{\mu}$ out of the covariant derivatives and into the Chern-Simons term $S_{\mathrm{CS}}$.

We are now ready to proceed with the semiclassical approximation. The Saddle Point Equations, or classical equations of motion are

$$
\left.\frac{\delta S_{\text {eff }}}{\delta a_{\mu}(z)}\right|_{\bar{a}}=0
$$

By varying $S_{\text {eff }}$ with respect to $a_{\mu}(z)$ we find

$$
\begin{aligned}
\left\langle j_{0}(z)\right\rangle_{F}= & -\theta[\langle\mathcal{B}(z)\rangle-\langle\tilde{B}(z)\rangle] \\
\left\langle j_{k}(z)\right\rangle_{F}= & +\theta \epsilon_{k l}\left(\left\langle\mathcal{E}_{l}(z)\right\rangle-\left\langle\tilde{E}_{l}\right\rangle\right) \\
& \left.-\theta \epsilon_{k l} \partial_{l}^{(z)} \int d^{3} z^{\prime} V\left(\mid \vec{z}-\vec{z}^{\prime}\right)\left(\theta\left(\langle\mathcal{B}-\tilde{B}\rangle\left(z^{\prime}\right)\right)-\bar{\rho}\right)\right\}
\end{aligned}
$$

where $\left\langle j_{\mu}(z)\right\rangle_{F}$ represents the expectation value of the charge and current of the equivalent fermion problem.

These equations have many possible solutions which include uniform (liquid) states, Wigner crystals, and non-uniform states with vortex-like configurations. We will only consider solutions with uniform particle density $\left(\left\langle j_{0}(z)\right\rangle=\bar{\rho}\right)$, i.e., the liquid phase solution, and no currents in the ground state. 
If the external electromagnetic fluctuation is assumed to have zero average, the only possible solutions of this type are

$$
\begin{aligned}
\langle\mathcal{B}\rangle & =-\frac{\bar{\rho}}{\theta} \\
\langle\overrightarrow{\mathcal{E}}\rangle & =0
\end{aligned}
$$

This is the average field approximation (AFA) which can be regarded as a mean field approximation. Eq. 21 shows that, for a translationally invariant ground state, the effect of the statistical gauge fields, at the level of the saddle-pointapproximation, is to change the effective flux experienced by the fermions. The total effective field is thus reduced from the value of the external field $B$ down to $B_{\text {eff }}=B+\langle\mathcal{B}\rangle=B-\frac{\bar{\rho}}{\theta}$. We want to find the ground state and the spectrum of low energy excitations of a system of $N$ (interacting) electrons in the presence of an external magnetic field of strength $B$. We will further assume that the linear size $L$ of the sample is such that a total of $N_{\phi}$ quanta of the magnetic flux are piercing the surface. In general, the filling fraction $\nu=\frac{N}{N_{\phi}}$ is not an integer. Notice that, as $B$ and $\rho$ are varied, the effective field $B_{\text {eff }}$ may either be parallel or anti-parallel to the external field $B$. Thus, we will not assume a particular sign for $B_{\text {eff }}$ although we will set $B>0$.

The uniform effective magnetic field $B_{\text {eff }}$, which solves Eq. 21, define a new set of effective Landau levels. Each level has a degeneracy equal to the total number of effective flux quanta $N_{\text {eff }}$ and the separation between levels is the effective cyclotron frequency $\omega_{c}^{\text {eff }}=\frac{e\left|B_{\text {eff }}\right|}{M c}$. Similarly, there is an effective cyclotron radius $\ell^{\text {eff }}$. It is easy to see that the uniform saddle-point state which satisfies Eq. 21 has a gap only if the effective field $B_{\text {eff }}$ experienced by the $N$ fermions is such that the fermions fill exactly an integer number $p$ of the effective Landau levels. This is precisely the point of view advocated by Jain: the FQHE is an IQHE of a system of electrons dressed by an even number of flux quanta. However, this condition cannot be met for arbitrary values of the filling fraction $\nu$ at fixed field ( or at fixed density). Let $N_{\phi}^{\text {eff }}$ denote the effective number of flux quanta piercing the surface after screening. It is given by

$$
\pm 2 \pi N_{\phi}^{\mathrm{eff}}=2 \pi N_{\phi}-\frac{\bar{\rho}}{\theta} L^{2}
$$

where the \pm sign stands for the case of an effective field parallel or antiparallel to $B$. Thus, the effective cyclotron frequency $\omega_{c}^{\text {eff }}$ is reduced from its free electron value of $\frac{e B}{M_{c}}$ down to $\omega_{c}^{\text {eff }}=\omega_{c}\left(1-\frac{\nu}{2 \pi \theta}\right)$. The effective cyclotron radius is given by $\ell^{\text {eff }}=\left(\ell / \sqrt{1-\frac{\nu}{2 \pi \theta}}\right)$ which is larger than the non-interacting value. Therefore, even though the bare Landau levels may be separated by a sizable Landau gap $\hbar \omega_{c}$, the effective Landau levels have the smaller gap $\hbar \omega_{c}^{\text {eff }}$. 
Substituting the value of $\theta$ in Eq. 22 we obtain

$$
\pm 2 \pi N_{\phi}^{\mathrm{eff}}=2 \pi N_{\phi}-2 \pi 2 s N .
$$

where $2 s$ is an even integer. The spectrum supported by this state has an energy gap if the $N$ fermions fill exactly $p$ of the Landau levels created by the effective field $B_{\text {eff }}$. In other words, the effective filling fraction is $\nu_{\text {eff }} \equiv \frac{N}{N_{\phi}^{\text {erf }}}=$ $p$. Using Eq. 23, we find that the filling fraction $\nu$ and the external magnetic field $B$ must satisfy

$$
\pm \frac{N}{p}=\frac{N}{\nu}-2 s N
$$

or, equivalently,the allowed filling fractions are

$$
\nu_{ \pm}(p, s)=\frac{p}{2 s p \pm 1}
$$

The allowed filling fractions $\nu_{ \pm}$can thus be arranged into families or hierarchies. As $p \rightarrow \infty$, the allowed fractions approach the limiting values $\lim _{p \rightarrow \infty} \nu_{ \pm}(p, s)=\frac{1}{2 s}$, with $\nu_{+}(p, s) \leq \frac{1}{2 s}$ while $\nu_{-}(p, s) \geq \frac{1}{2 s}$. For the special case $s=1$, the limiting value is $\frac{1}{2}$. Since $\nu_{+}(p, 1) \leq \frac{1}{2}$ and $\nu_{-}(p, 1) \geq \frac{1}{2}$, the sequence $\nu_{+}(p, 1)$ is said to represent electron-like FQH states whereas the mirror sequence $\nu_{-}(p, 1)$ is said to represent hole-like FQH states. The effective Landau gap for these solutions is

$$
\hbar \omega_{c}^{\mathrm{eff}}=\frac{\hbar \omega_{c}}{2 s p \pm 1}
$$

which is small if either $p$ or $s$ are large. Thus, the energy to excite a fermion is $\hbar \omega_{c}^{\text {eff }}$ and it is considerably smaller than the bare free-particle value $\hbar \omega_{c}$.

The states are thus parametrized by two integers $p$ ( the number of filled effective Landau levels of the effective field) and $2 s$ ( the number of flux quanta carried by each fermion), and by a sign. It is trivial to see that the Laughlin sequence is an obvious solution of Eq. 25 for $p=1$ and $2 s=m-1$. The effective fermions thus fill up exactly one Landau level and $\theta$ has to be chosen to be $\frac{1}{\theta}=2 \pi(m-1)$. This is Jain's result. At this mean-filled level the wave function is the Slater determinant for one filled Landau level $\chi_{1}$ of Eq. 2. We will show below that the additional factor $\prod_{i<j}\left(z_{i}-z_{j}\right)^{m-1}$ is due to fluctuations. For general $p$ and $s$, these are the allowed states in Jain's hierarchy 20. Please notice that, unless $s=0$, all states in these hierarchies have $\nu_{ \pm}(p, s) \leq 1$. For $s=0$ (i.e., no flux attachement) $\nu=p$ and we reproduce the integer $\mathrm{QH}$ states. Thus, only primary FQH states are generated. States characterized by other fractions can be constructed by means of the conventional scheme of 
condensing quasiparticles or quasiholes on top of these sequencies. We will see below that, as expected, the quasiparticles and quasiholes of the FQH states in these hierarchies are anyons. However, it is unclear how many of these higher level hierarchical states are energetically stable (or accessible).

The saddle-point approximation yields a very simple spectrum which consists just in the single and many-particle excitations of fermions in the effective field $B_{\text {eff }}$. The single-particle gap is equal to $\hbar \omega_{c}^{\text {eff }}=\hbar \omega_{c} /(2 s p \pm 1)$. Thus, at fixed $B$, the single particle energy gaps become smaller as the index $p$ increases and vanish as $p \rightarrow \infty$. In this limit the hierarchical states converge to the fractions $\nu=\frac{1}{2 s}$. For these fractions the system is compressible, $i . e$., there is no energy gap in its spectrum, and the perturbation expansion breaks down. The breakdown is signalled by infrared divergent contributions at every order of the expansion at low temperatures. The case of the $\frac{1}{2 s}$ states was analyzed by Halperin, Lee and Read 16 . Elsewhere in this volume, Steve Simon presents a detailed discussion of this important case.

At this level of the SPE, the Coulomb energy does not enter explicitly in the effective single particle gaps. However, their existence is a physical consequence of the existence of Coulomb interactions. Moreover, in the strong field limit $B \rightarrow \infty$, both the bare and the effective Landau gaps diverge. This is clearly incorrect since, as $B$ increases the states of the system should be made almost completely of states only in the lowest Landau level. In the strong field limit, the Coulomb energy alone should determine the numerical value of the gaps. The origin of this difficulty is that the SPE (or the RPA) is formally correct in the small field limit and the states exhibiting the FQHE are all in the large field limit. Thus, we should expect that the higher order correction in the SPE should not only correct the numerical value of the gaps but also lead to a finite limit in the strong field regime. These corrections have not been calculated and their properties are not yet understood. The same considerations apply to the spectrum of collective excitations discussed in Section 3.1. However, it is important to stress that the SPE (with gaussian corrections) yields a set of states with the correct quantum numbers even though the energies are not correct. In particular we will show below that, once gaussian fluctuation are accounted for, these single particle states have the correct charge and statistics (although the wrong energy).

The saddle-point equations, Eq. 20, have a host of non-uniform solutions which have finite energy. They can be viewed as soliton or vortex solutions. Our construction is very close in spirit to the picture of the quasihole presented by Laughlin 17. We will only discuss here the qualitative features of these solutions. For the sake of simplicity, we will only study the case of fermions in the Laughlin $\frac{1}{m}$ sequence. Recall that this sequence is represented by the 
solution with $p=1$ and $m=2 s+1$. The uniform state was constructed by filling up the lowest effective Landau level. Let us consider the state which results from removing a fermion from the single-particle state, centered around the origin $z=0$ and with lowest angular momentum, and placing it on the first unoccupied angular momentum state. Physically, this new state lies on the outer edge of the system. For an uniform effective field, this state does not exist. But, if the effective field is increased at the origin by an amount equal to one flux quantum, the angular momentum of all its eigenstates is raised by one whole unit. The radius $R_{N}$ of the droplet with $N$ particles swells to a new value $R_{N}+\delta R$ large enough to include a new cyclotron orbit. Qualitatively, a quasihole localized at $z_{0}$ has the mean-field wave function $\Psi_{h}=\Psi_{h}\left(z_{0},\left\{z_{j}\right\}\right)$ given by

$$
\Psi_{h}\left(z_{0},\left\{z_{j}\right\}\right)=\prod_{i=1}^{N}\left(z_{i}-z_{0}\right) \chi_{1}\left(\left\{z_{j}\right\}\right) .
$$

where $\chi_{1}\left(\left\{z_{j}\right\}\right)$ is the wave function for $N$ fermions occupying the lowest Landau level. Notice that this wave function differs from the Laughlin state for the quasihole by the prefactor $\prod_{i<j}\left(z_{i}-z_{j}\right)^{m-1}$. Indeed, this prefactor is also missing in the mean-field wave function for the ground state. In our picture, both prefactors arise from fluctuations which attach fluxes to the particles. The quasihole wave function of Eq. 27 is an approximation valid in the limit $\left|z_{i}-z_{0}\right| \gg \ell^{\text {eff }}$. From Eq. 20 it is easy to see that the mean-field excitation energy of the quasihole $\varepsilon_{h}$ is given approximately by the Coulomb energy $V\left(\ell^{\mathrm{eff}}\right)$.

\subsection{The role of the Gaussian fluctuations}

We consider now the role of the gaussian fluctuations around the uniform classical solutions discussed in Section 2.1. We begin by considering the effective action of Eq. 18. Let $\tilde{a}_{\mu}(x)$ denote the fluctuations of the statistical vector potential $a_{\mu}, i$. e., we set $a_{\mu} \rightarrow\left\langle a_{\mu}\right\rangle+\tilde{a}_{\mu}$. The effective action of Eq. 18 can be expanded in a series in powers of the fluctuations. We will be interested only in keeping just up to quadratic terms in the fluctuations. In the language of Feynman diagrams, we are summing up all the one-loop bubble contributions. Thus, the effective action at the quadratic level involves the linear response kernels (evaluated in the RPA) for a system of fermions in an external static uniform magnetic field $B_{\text {eff }}$ with an integer number of filled effective Landau levels. As usual, the linear terms are cancelled if the saddle-point equations are satisfied. 
At the quadratic (gaussian) level the effective action has the form

$$
S^{(2)}=\frac{1}{2} \int d^{3} x d^{3} y \tilde{a}_{\mu}(x) \Pi^{\mu \nu}(x, y) \tilde{a}_{\nu}(y)+S_{\mathrm{CS}}\left(\tilde{a}_{\mu}-\tilde{A}_{\mu}\right)+S_{\mathrm{int}}^{(2)}\left(\tilde{a}_{\mu}-\tilde{A}_{\mu}\right) .
$$

where

$$
S_{\text {int }}^{(2)}\left(\tilde{a}_{\mu}-\tilde{A}_{\mu}\right)=-\frac{\theta^{2}}{2} \int d^{3} x d^{3} y[\tilde{\mathcal{B}}(x)-\tilde{B}(x)] V(\vec{x}-\vec{y})[\tilde{\mathcal{B}}(y)-\tilde{B}(y)]
$$

Notice that the only approximation used in Eq. 28 is in the first term which follows from the expansion of the fermion determinant in powers of $\tilde{a}_{\mu}$. The remaining terms are exact.

Eq. 28 holds provided that the non-quadratic dependence in the fluctuating part of the statistical vector potentials $\tilde{a}_{\mu}$ is small. Recall that these non-quadratic terms result from expanding the (logarithm) of the fermion determinant in powers of the fluctuations around the average field approximation. The kernels that enter in the expressions for these terms are (connected) current correlation functions (or response functions) of the mean field theory. Thus, the tensor $\Pi_{\mu \nu}^{(0)}$ is the polarization tensor of the equivalent fermion problem at the mean field level, and it is obtained by expanding the fermion determinant up to quadratic order in the statistical gauge field

$$
\begin{aligned}
\mathcal{Z}_{0}\left[A_{\mu}\right] & \equiv \operatorname{Det}\left[i D_{0}+\mu+\lambda+\frac{1}{2 M} \vec{D}^{2}\right] \\
& =\mathcal{Z}[0] \exp \left\{\frac{i}{2} \int d^{D} x \int d^{D} y A_{\mu}(x) \Pi_{\mu \nu}^{(0)}(x, y) A_{\nu}(y)+\ldots\right\}
\end{aligned}
$$

The tensor $\Pi_{\mu \nu}^{(0)}$ should not be confused with the true electromagnetic polarization tensor which measures the response of the whole system to a weak electromagnetic field. We will come back to this issue in the next Section.

It is straightforward to derive an expression for $\Pi_{\mu \nu}^{(0)}$ in terms of the oneparticle Green functions $G(x, y)$

$$
G(x, y)=<x\left|\frac{1}{i D_{0}+\mu+\frac{1}{2 M} \vec{D}^{2}[<A>]}\right| y>.
$$

The components of the polarization tensor $\Pi_{\mu \nu}^{(0)}(x, y)$ are $(\hbar=1)$

$$
\begin{aligned}
\Pi_{00}^{(0)}(x, y) & =i G(x, y) G(y, x) \\
\Pi_{0 j}^{(0)}(x, y) & =\frac{1}{2 M}\left\{G(x, y) D_{j}^{y} G(y, x)-G(y, x) D_{j}^{y^{\dagger}} G(x, y)\right\}
\end{aligned}
$$




$$
\begin{aligned}
\Pi_{j 0}^{(0)}(x, y) & =+\frac{1}{2 M}\left\{-G(x, y) D_{j}^{x \dagger} G(y, x)+G(y, x) D_{j}^{x} G(x, y)\right\} \\
\Pi_{j k}^{(0)}(x, y) & =\frac{i}{M} \delta(x-y) \delta_{j k} G(x, y)+ \\
- & \frac{i}{4 M^{2}}\left(D_{j}^{x} G(x, y)\right)\left(D_{k}^{y} G(y, x)\right)-\frac{i}{4 M^{2}}\left(D_{j}^{x \dagger} G(y, x)\right)\left(D_{k}^{y \dagger} G(x, y)\right)+ \\
+ & \left.\frac{i}{4 M^{2}} G(y, x)\right)\left(D_{j}^{x} D_{k}^{y \dagger} G(x, y)\right)+\frac{i}{4 M^{2}}\left(D_{j}^{x \dagger} D_{k}^{y} G(y, x)\right) G(x, y) .
\end{aligned}
$$

It can be shown that the effective action of Eq. 30 is gauge-invariant and that, in consequence, $\Pi_{\mu \nu}^{(0)}$ is transverse, $i . e$. ,

$$
\partial_{\mu}^{x} \Pi_{\mu \nu}^{(0)}(x, y)=0 .
$$

In reference日 1 we give detailed expressions for the various components of $\Pi_{\mu \nu}^{(0)}$. In particular we show that the action is gauge-invariant and that $\Pi_{\mu \nu}^{(0)}$ is transverse, albeit only in a weak sense $i$. e., as a distribution. Eq. (32) agrees with the results of Randjbar-Daemi, Salam and Strathdee 18 but disagrees with the calculation of $\Pi_{\mu \nu}^{(0)}$ by Chen et. al. 19 .

From the expressions for the components of $\Pi_{\mu \nu}^{(0)}$ in referencel, we see that this tensor is analytic in $\frac{\vec{Q}^{2}}{B_{\text {eff }}}$ and that it has simple poles at $\omega=k \bar{\omega}_{c}$ (with $k$ an integer), where $\bar{\omega}_{c} \equiv \omega_{c} /(2 s p+1)$ is the cyclotron frequency associated with the effective magnetic field $B_{\text {eff }}$. As a result, $\Pi_{\mu \nu}^{(0)}$ has a gradient expansion in powers of the inverse of the effective magnetic field $\frac{1}{B_{\text {eff }}}$, or equivalently, in powers of the inverse of the external magnetic field $\frac{1}{B}$. In fact, the dimensionless parameter of this expansion is $\frac{\vec{Q}^{2}}{B}$ (we are working in a system of units such that $\hbar=c=e=1$ ). It also turns out that, within this approximation, the limits of $B \rightarrow \infty$ and $M \rightarrow 0$ are not equivalent (see the explicit form of $\Pi_{\mu \nu}^{(0)}$ given in reference国).

The non-quadratic terms in $\tilde{a}_{\mu}$ in the effective action are of the form

$$
S_{\mathrm{eff}}=S^{(2)}\left(\tilde{a}^{\mu}, \tilde{A}^{\mu}\right)+\frac{1}{3 !} \int d^{3} x d^{3} y d^{3} z \tilde{a}^{\mu} \tilde{a}^{\nu} \tilde{a}^{\lambda} \Pi_{\mu \nu \lambda}^{(0)}(x, y, z)+\ldots
$$

where the kernel $\Pi_{\mu \nu \lambda}^{(0)}(x, y, z)$ represents a three-point current correlation function in the mean field theory. Thus, in the language of Feynman diagrams, while $\Pi_{\mu \nu}^{(0)}(x, y)$ can be viewed as a fermion bubble with two amputated external collective mode lines, $\Pi_{\mu \nu \lambda}^{(0)}(x, y, z)$ again has one fermion loop tied to 
three amputated external collective mode lines $\tilde{a}_{\mu}$. Each one of these nonquadratic kernels have the same gauge invariance ( $i$. e., transversality) and analytic properties as the gaussian (or RPA) kernel. In particular, this means that, in momentum and frequency space, these kernels must be a linear combination of tensors (of the appropriate rank) which have the correct transversality properties, times a set of functions which are analytic in $\vec{Q}^{2}$ and have poles at frequencies equal to an integer multiple of the effective cyclotron frequency. Therefore, the non-quadratic terms necessarily have powers of $\frac{\vec{Q}^{2}}{B_{\text {eff }}}$ (for each one of the external momenta and frequency entering the fermion loop) which are higher than the ones found at the quadratic level. Since the mean field theory has an integer number of filled Landau levels, the energy denominators of the kernels do not change this counting in powers of $\frac{\vec{Q}^{2}}{B_{\text {eff }}}$. In conclusion, the expansion of the fermion determinant, and hence of the effective action, is actually an expansion in powers of $\frac{\vec{Q}^{2}}{B_{\text {eff }}}$, or equivalently, in powers of $\frac{\vec{Q}^{2}}{B}$. However, an expansion in powers of $\frac{\vec{Q}^{2}}{B}$ is also a gradient expansion. Thus, the gradient expansion and the semiclassical expansion mix and are not independent from each other.

The semiclassical expansion is obtained according to the following rules. The propagator for the fluctuations, which represent collective modes, is the inverse of the kernel of the gaussian action. Since the pair potential enters only through the propagator for the fluctuations, the perturbation theory is not an expansion in the powers of the pair interaction. From this point of view, this expansion is very different from conventional expansions around the Hartree and Hartree-Fock approximations. The vertices of the expansion are the kernels for the non-quadratic terms. This expansion lacks a natural small parameter ( $i$. e., a coupling constant) and it should be regarded, like all semiclassical expansions, as an expansion in the number of fermion loops (i. e., RPA plus corrections). One should keep in mind, however, our previous discussion on its exactness in powers of $\frac{\vec{Q}^{2}}{B}$. In what follows we will make extensive use of the formal properties of this expansion.

We remark here that, unlike other mean field approaches to conventional many body systems (such as Hartree-Fock), the gaussian corrections of this theory must alter the qualitative properties of the state described by the AFA. The reason is that the AFA violates explicitly space-time symmetries, such as Galilean invariance (more generally, magnetic invariance) which, for translationally invariant systems, must remain unbroken and unchanged. Thus the center of mass of the system must execute a cyclotron-like motion at, exactly, the cyclotron frequency of non interacting electrons in the full external magnetic field, as demanded by Kohn's theorem20. A naïve application of the AFA 
would suggest that the cyclotron frequency is renormalized downwards since the effective field seen by the composite fermions is smaller than the external field $B$. Hence, the magnetic algebra may appear to have changed. We will see below that the gaussian fluctuations yield the correct cyclotron frequency and, thus, restore the correct magnetic algebra.

\section{Response functions and spectrum of collective excitations}

The physical systems which exhibit the FQHE present a very rich response to external electromagnetic perturbations. While some of the observed phenomena, such as cyclotron resonance, can be understood in terms of simple global motions of the center of mass under the combined influence of electric and magnetic fields, the spectrum of collective excitations is certainly determined by the interactions. Given the unusual features of the Laughlin states and its generalizations, it is expected that the same features should largely determine the behavior of the collective modes too. We will now use the fermion field theory described in Section 2, to study the collective excitations of the fully polarized FQHE states in the sequence $1 / \nu= \pm 1 / p+2 s$ (where $p$ and $s$ are two positive integers). While the average field approximation is certainly very appealing, it has the serious problem that it breaks a number of space-time symmetries quite explicitly. In particular, it breaks both Galilean and Magnetic invariance. It turns out that the leading quantum fluctuations around this state, restore these symmetries, in the uniform $\vec{Q} \rightarrow 0$ limit, already at the gaussian level. Indeed, we find that the quadratic or gaussian level of the semiclassical expansion gives the correct value of the Hall conductance of the system, and that the leading order of the density correlation function saturates the $f$-sum rule. This is an essential result to show that the absolute value squared of the wave function of all the (incompressible) liquid states has the Laughlin form at very long distances, in the thermodynamic limit (see Section 5).

\subsection{Electromagnetic response functions for the FQHE}

The effective action for the fluctuations of the Chern-Simons gauge field $\left(\tilde{a}_{\mu}\right)$, at the Gaussian level of our approximation, is given by

$$
\begin{aligned}
S^{(2)}\left(\tilde{a}^{\mu}, \tilde{A}^{\mu}\right) & =\frac{1}{2} \int d^{3} x d^{3} y \tilde{a}^{\mu}(x) \Pi_{\mu \nu}^{(0)}(x, y) \tilde{a}^{\nu}(y)+\frac{\theta}{4} S_{\mathrm{cs}}\left(\tilde{a}_{\mu}-\tilde{A}_{\mu}\right) \\
& -\frac{\theta^{2}}{2} \int d^{3} x d^{3} y[\tilde{\mathcal{B}}(x)-\tilde{B}(x)] V(x-y)[\tilde{\mathcal{B}}(y)-\tilde{B}(y)]
\end{aligned}
$$


Since $S^{(2)}$ is quadratic in $\tilde{a}_{\mu}$, we can integrate out this field and obtain the effective action for the electromagnetic fluctuations $\tilde{A}_{\mu}, S_{\text {eff }}^{\text {em }}\left(\tilde{A}_{\mu}\right)$. We will use this effective action to calculate the full electromagnetic response functions at the gaussian level. Since this calculation is based on a one loop effective action for the fermions (i.e., a sum of fermion bubble diagrams), this approximation amounts to a random phase correction to the average field approximation.

In order to integrate out the statistical gauge field $\tilde{a}_{\mu}$ we must fix the gauge. The electromagnetic effective action, being gauge invariant, is independent of the choice of gauge for the statistical gauge fields in the path integral. We fix the gauge $\partial_{\mu} \tilde{a}^{\mu}=\alpha$ (where $\alpha$ is an arbitrary real number) using the standard Faddeev-Popov procedure 9 . The result is explicitly gauge invariant and all dependence on the parameter $\alpha$ cancels out. At the one-loop level (governed by the effective action of Eq. 35) we need to know the inverse of the polarization tensor of the equivalent fermion problem, $\Pi_{\mu \nu}^{(0)}$. In reference 1 , we showed that $\Pi_{\mu \nu}^{(0)}$ can be written in terms of three gauge invariant tensors, an $\vec{E}^{2}$ term, a $\vec{B}^{2}$ term, and a Chern-Simons term. These three tensors plus $B \vec{\nabla} \cdot \vec{E}$ and a gauge fixing term (such as $\frac{1}{2 \alpha}\left(\partial_{\mu} \tilde{a}^{\mu}\right)^{2}$ which corresponds to the Landau-Lorentz gauge if $\alpha \rightarrow 0$ ), close an algebra that can be used to invert the polarization tensor and to calculate explicitly the electromagnetic response functions.

After integrating out the statistical gauge field in Eq. 35, the effective action for the electromagnetic fluctuations $\tilde{A}_{\mu}$ turns out to be

$$
\mathcal{S}_{\text {eff }}^{\mathrm{em}}\left(\tilde{A}_{\mu}\right)=\frac{1}{2} \int d^{3} x d^{3} y \tilde{A}_{\mu}(x) K^{\mu \nu}(x, y) \tilde{A}_{\nu}(y)
$$

Here $K^{\mu \nu}$ is the electromagnetic polarization tensor. It measures the linear response of the system to a weak electromagnetic perturbation. Its components can be written in momentum space as follows

$$
\begin{aligned}
K_{00} & =\vec{Q}^{2} K_{0}(\omega, \vec{Q}) \\
K_{0 j} & =\omega Q_{j} K_{0}(\omega, \vec{Q})+i \epsilon_{j k} Q_{k} K_{1}(\omega, \vec{Q}) \\
K_{j 0} & =\omega Q_{j} K_{0}(\omega, \vec{Q})-i \epsilon_{j k} Q_{k} K_{1}(\omega, \vec{Q}) \\
K_{i j} & =\omega^{2} \delta_{i j} K_{0}(\omega, \vec{Q})-i \epsilon_{i j} \omega K_{1}(\omega, \vec{Q})+\left(\vec{Q}^{2} \delta_{i j}-Q_{i} Q_{j}\right) K_{2}(\omega, \vec{Q})
\end{aligned}
$$

${ }^{a}$ To be more precise, whenever we have had to fix the gauge we have adopted Feynman's method of averaging over gauges, with $\alpha$ being the width of the distributions. This is a standard procedure which is reviewed in many textbooks (see, for instance, reference 21). 
where the functions $K_{l}(\omega, \vec{Q})(l=0,1,2)$ are given by

$$
\begin{aligned}
K_{0}(\omega, \vec{Q}) & =-\theta^{2} \frac{\Pi_{0}}{D(\omega, \vec{Q})} \\
K_{1}(\omega, \vec{Q}) & =\theta+\theta^{2} \frac{\left(\theta+\Pi_{1}\right)}{D(\omega, \vec{Q})}+\theta^{3} \tilde{V}(\vec{Q}) \vec{Q}^{2} \frac{\Pi_{0}}{D(\omega, \vec{Q})} \\
K_{2}(\omega, \vec{Q}) & =-\theta^{2} \frac{\Pi_{2}}{D(\omega, \vec{Q})}+\frac{\tilde{V}(\vec{Q})\left(\omega^{2} \Pi_{0}{ }^{2}-\Pi_{1}^{2}\right)}{D(\omega, \vec{Q})}+\frac{\tilde{V}(\vec{Q}) \vec{Q}^{2} \Pi_{0} \Pi_{2}}{D(\omega, \vec{Q})}
\end{aligned}
$$

and

$$
D(\omega, \vec{Q})=\Pi_{0}^{2} \omega^{2}-\left(\Pi_{1}+\theta\right)^{2}+\Pi_{0}\left(\Pi_{2}-\theta^{2} \tilde{V}(\vec{Q})\right) \vec{Q}^{2}
$$

The coefficients $\Pi_{l}(l=0,1,2)$ are functions of $\omega$ and $\vec{Q}$, and are given explicitly in reference $\vec{E} . \tilde{V}(\vec{Q})$ is the Fourier transform of the interparticle pair potential. As we mentioned before, we needed to include a gauge fixing term to be able to compute the functional integral in Eq. 35. But at the end of the calculation all the terms which contain the gauge fixing coefficient $(\alpha)$ cancel each other, and the final result for the response functions is, as it must be, gauge invariant. The other tensor that we have introduced to make the calculations, $B \vec{\nabla} \cdot \vec{E}$, is not present in the final answer either.

We want to stress here that the thermodynamic limit is crucial for the accuracy of our results. Notice first that in the electromagnetic effective action of Eq. 36 we are neglecting higher order response functions, i. e., correlation functions of three or more currents or densities. We have shown in Section 2.2 that these higher order correlation functions have higher order powers of $\frac{Q^{2}}{B}$ than the quadratic term. Strictly speaking, these terms are not neglectible for a finite system because, in this case, there is a minimum value that the momentum can take, determined by the linear size of the system $L, i$. e., $|\vec{Q}|>\frac{1}{L}$. But in the thermodynamic limit, $L \rightarrow \infty$ and the momentum can go to zero. In other words, only for an infinite system one is allowed to keep only the quadratic term in the electromagnetic action, Eq. 36, and to neglect the higher order correlation functions.

The electromagnetic response functions determined by $K_{\mu \nu}$ have the following properties:

i) The polarization tensor at mean field level, $\Pi_{\mu \nu}^{(0)}$, has poles at every value of the effective cyclotron frequency $\left(\bar{\omega}_{c} \equiv \frac{B_{\text {eff }}}{M}\right)$. This corresponds to the physical picture, at mean field level, of an IQHE of the bound states in the presence of a partially screened external magnetic field, $B_{\text {eff }}$. Once we take into 
account the Gaussian fluctuations, it is easy to prove that all this poles that are present in the numerator and the denominator of the $K_{\mu \nu}$ components through the $\Pi_{j}$ 's, cancel out, and the poles of the response functions are determined only by the zeroes of their denominator, $D(\omega, \vec{Q})$. In other words, the collective excitations of this system will be determined only by the zeroes of $D(\omega, \vec{Q})$.

ii) The leading order term in $\vec{Q}^{2}$ of the $K_{00}$ component of the polarization tensor saturates the $f$-sum rule.

iii) The gaussian fluctuations of the statistical gauge field are responsible for the FQHE. In particular, the gaussian corrections yield the exact value for the Hall conductance.

In the remaining of this section we will discuss these properties and their experimentally accessible consequences in detail.

\subsection{The Spectrum of Collective Excitations}

For simplicity, we give here the zeroes of $D(\omega, \vec{Q})$ for the case of $p=1$ filled effective Landau levels. The (more tedious) case of general $p$ can be studied by straightforward application of the same methods 22 .

In the case $p=1$ the filling fraction is $\nu=\frac{1}{m}$, where $m=1+2 s, i$. e., the Laughlin sequence. We find that there is a family of collective modes whose zero-momentum gap is $k \bar{\omega}_{c}$, where $k$ is an integer number different from 1 and $m$, and whose dispersion curve $\omega_{k}(\vec{Q})$ is

$$
\omega_{k}(\vec{Q})=\left[\left(k \bar{\omega}_{c}\right)^{2}+\left(\frac{\vec{Q}^{2}}{2 B_{\mathrm{eff}}}\right)^{k-1} \bar{\omega}_{c}^{2} \frac{2 k(m-1)(k-1)}{(k-1) !(k-m)}\right]^{\frac{1}{2}}
$$

The residue in $K_{00}$ corresponding to this pole is

$$
\operatorname{Res}\left(K_{00}, \omega_{k}(\vec{Q})\right)=-\vec{Q}^{2} \omega_{c} \frac{\nu}{2 \pi}\left(\frac{\vec{Q}^{2}}{2 B_{\mathrm{eff}}}\right)^{k-1} \times \frac{2 k(m-1)(k-1)}{(k-1) !(k-m)\left(k^{2}-m^{2}\right)}
$$

The cases $k=1, m$ have to be treated separately. In general, we find that there is no mode with a zero momentum gap at $\bar{\omega}_{c}$. Instead, at $\vec{Q}=0$, there is a doubly degenerate mode with a gap at $\omega_{c}$. This degenerate cyclotron mode can be viewed as the mixing of the modes with $k=1$ and with $k=m$. Thus, the mode with $k=1$ has been "pushed up" to the cyclotron frequency (at $\vec{Q}=0$ ). For $\vec{Q} \neq 0$, the degeneracy is lifted and these two modes have different dispersion curves.

For the special case of $\nu=\frac{1}{3}, i$. e., $m=3$, this effect is particularly important. The dispersion relations for the cyclotron modes are given by

$$
\omega_{ \pm}(\vec{Q})=\left[\omega_{c}^{2}+\left(\frac{\vec{Q}^{2}}{2 B_{\mathrm{eff}}}\right) \frac{\bar{\omega}_{c}^{2}}{2} \alpha_{ \pm}\right]^{\frac{1}{2}}
$$


where

$$
\alpha_{ \pm}=8+\frac{2 M \tilde{V}(0)}{2 \pi} \pm\left(\left(8+\frac{2 M \tilde{V}(0)}{2 \pi}\right)^{2}+288\right)^{\frac{1}{2}}
$$

The residues corresponding to these poles are

$$
\operatorname{Res}\left(K_{00}, \omega_{ \pm}(\vec{Q})\right)=-\vec{Q}^{2} \omega_{c} \frac{\nu}{2 \pi}\left(1+\frac{288}{\alpha_{ \pm}^{2}}\right)^{-1}
$$

For $\nu=\frac{1}{m}, m \geq 5$, the collective modes whose zero-momentum gap is the cyclotron frequency, $\omega_{c}$, are

$$
\omega_{+}(\vec{Q})=\left[\omega_{c}^{2}+\left(\frac{\vec{Q}^{2}}{2 B_{\mathrm{eff}}}\right) \bar{\omega}_{c}^{2} \times\left(4 \frac{(m-1)}{(m-2)}+\frac{2 M \tilde{V}(0)}{2 \pi}\right)\right]^{\frac{1}{2}}
$$

with residue

$$
\operatorname{Res}\left(K_{00}, \omega_{+}(\vec{Q})\right)=-\vec{Q}^{2} \omega_{c} \frac{\nu}{2 \pi}
$$

The other cyclotron mode has the dispersion

$$
\omega_{-}(\vec{Q})=\left[\omega_{c}^{2}-\left(\frac{\vec{Q}^{2}}{2 B_{\text {eff }}}\right)^{m-2} \bar{\omega}_{c}^{2} \frac{4 m^{2}(m-1)^{2}}{(m-1) !}\left(4 \frac{(m-1)}{(m-2)}+\frac{2 M \tilde{V}(0)}{2 \pi}\right)^{-1}\right]^{\frac{1}{2}}
$$

with residue

$$
\begin{aligned}
& \operatorname{Res}\left(K_{00}, \omega_{-}(\vec{Q})\right)= \\
& \quad-\vec{Q}^{2} \omega_{c} \frac{\nu}{2 \pi}\left(\frac{\vec{Q}^{2}}{2 B_{\mathrm{eff}}}\right)^{m-3} \frac{4 m^{2}(m-1)^{2}}{(m-1) !}\left(4 \frac{(m-1)}{(m-2)}+\frac{2 M \tilde{V}(0)}{2 \pi}\right)^{-2}
\end{aligned}
$$

The above results are valid only if the pair potential $\tilde{V}(\vec{Q})$, has a gradient expansion in powers of $\vec{Q}, i$. e., for short range interactions. $\tilde{V}(0)$ stands for the leading order term in that expansion.

If the pair potential has the Coulomb form, i. e., $\tilde{V}(\vec{Q})=\frac{2 \pi q^{2}}{|\vec{Q}|}$ in two spacial dimensions, both, the dispersion relations with zero-momentum gap at the cyclotron frequency and their residues get modified. The expressions valid in this case are, for any allowed value of $m$

$$
\omega_{+}(\vec{Q})=\left[\omega_{c}^{2}+\frac{|\vec{Q}|}{2 B_{\text {eff }}} \bar{\omega}_{c}^{2} 2 M q^{2}\right]^{\frac{1}{2}}
$$

with the same residue given by Eq. 46, and

$$
\omega_{-}(\vec{Q})=\left[\omega_{c}^{2}-\frac{|\vec{Q}|^{2 m-3}}{\left(2 B_{\mathrm{eff}}\right)^{m-2}} \bar{\omega}_{c}^{2} \frac{4 m^{2}(m-1)^{2}}{2 M q^{2}(m-1) !}\right]^{\frac{1}{2}}
$$


with residue

$$
\operatorname{Res}\left(K_{00}, \omega_{-}(\vec{Q})\right)=-\vec{Q}^{2} \omega_{c} \frac{\nu}{2 \pi} \frac{|\vec{Q}|^{2(m-2)}}{\left(2 B_{\text {eff }}\right)^{m-3}} \times \frac{4 m^{2}(m-1)^{2}}{\left(2 M q^{2}\right)^{2}(m-1) !}
$$

In this section we have found the spectrum of collective excitations for some values of the filling fraction. Our results are a generalization of the work of Kallin and Halperin 24 who studied the spectrum of collective modes for the integer quantum Hall effect within the RPA. We find a family of collective modes with dispersion relations whose zero-momentum gap is $k \bar{\omega}_{c}$, where $k$ is an integer number different from 1 and $m$. When $k=m, i$. e., the zeromomentum gap is the cyclotron frequency, there is a splitting in the dispersion relation for finite wavevector. One of these solutions, $\omega_{-}$, has negative slope for small values of $\vec{Q}$. Therefore, there must be a roton minimum at some finite value of the wavevector. Since our results are accurate only for small $\vec{Q}$, our dispersion curves do not apply close to the roton minimum. Nevertheless, this mode is expected to become damped due to non-quadratic interactions among the collective modes. On the other hand, the collective mode with lowest energy, which has $k=2$, is stable (at least for reasonably small wavevectors) and, at small wavectors, it disperses downwards in energy. This behavior suggests that there should be a magnetoroton minimum for this mode. This result is consistent with the work of Girvin et al. 25, where the authors used the single mode approximation to obtain the lowest collective mode in the lowest Landau level (the intra mode) for the states in the Laughlin sequence. The generalization of these results to other states of Jain's hierarchy $(p \neq 1)$ has been given in reference 2 . Our results coincide, in the small momentum limit, with the ones obtained by Simon et al. 23 more recently. The splitting of the cyclotron mode for $\nu=1 / 3$ is a little puzzling. It only happens for $\nu=1 / 3$ and for short range interactions. In all other cases, only the residue for one of the two cyclotron modes is proportional to $\vec{Q}^{2}$. Standard lore has it that Kohn's theorem demands that there should be one and only one mode converging to the cyclotron frequency as $\vec{Q}^{2} \overrightarrow{0}$ with residue proportional to $\vec{Q}^{2}$. Zhang has emphasized this point recently 26 . It is generally assumed that Kohn's theorem is valid even at non-zero wavevectors and that it requires the existence of only one mode with residue proportional to $\vec{Q}^{2}$ converging to $\omega_{c}$. However, at non-zero wave vectors, these arguments make the unstated assumption of the analyticity of the current operators on the wavevectors. While this may well be correct, it is an additional assumption and it does deserve closer scrutiny. The results from our theory do indeed predict the existence of only one mode at $\omega_{c}$ with residue proportional to $\vec{Q}^{2}$, which is the statement of Kohn's theorem. And, also, for all filling fractions and for all pair potentials (except $\nu=1 / 3$ 
and short range interactions) we do find only one mode with residue $\vec{Q}^{2}$ even at non-zero wavevectors. The case $\nu=1 / 3$ and short range interactions appears to be exceptional in that we find two modes which coalesce at the cyclotron frequency as $\vec{Q}^{2} \rightarrow 0$. But both of these modes have residue proportional to $\vec{Q}^{2}$, with different amplitude, and together they satisfy the sum rulet. While it is possible that the non-gaussian corrections may change this result since, in a sense, these are subleading pieces in $\vec{Q}^{2}$, these non-gaussian corrections are expected to be very small at small wavevectors.

We close this section with a few comments on the validity of this spectrum of collective modes beyond the semiclassical approximation. Primarily we have to consider the physics at moderately large wavevectors and the (expected) effects of non-gaussian corrections. At the gaussian (RPA) level we found a family of collective modes which, for sufficiently small momentum, are infinitely long lived (i.e., the response functions have delta-function sharp poles at their location). These modes represent charge-neutral bound states. It is in principle possible that, for $\vec{Q}$ sufficiently large, these modes should become damped. The threshold should occur when the energy of the collective mode becomes equal to the energy necessary to create the lowest available two-particle state: a quasiparticle-quasihole pair. In the AFA, the energy of a pair is equal to $\bar{\omega}_{c}$. Gaussian fluctuations are expected to renormalize this energy and to give it a momentum dependence. This is in principle calculable with the methods described here 23. Non-gaussian corrections to the RPA are also expected to give a finite width to (presumably) all the collective modes but the lowest one. This is so because the corrections to the semiclassical approximation are due to effective vertices (due to virtual quasiparticle-quasihole pairs) which couple the various collective modes and, thus, induce the higher energy modes to decay down into the lower modes. However, by gauge invariance, these vertices have a momentum dependence and should vanish as $\vec{Q} \rightarrow 0$. Thus, the width of the higher energy modes goes to zero as $\vec{Q} \rightarrow 0$ and these modes only become sharp at $\vec{Q}=0$. But at $\vec{Q}=0$ the only accessible mode is the cyclotron mode (the other modes have a vanishingly small spectral weight). These arguments strongly suggest that the only truly sharp mode, at $\vec{Q}=0$, is the cyclotron mode, which is required to be stable by Kohn's theorem 20. Since the modes with zero momentum gap at $k \bar{\omega}_{c}, k \geq 3$, are not the collective modes with lowest energy, it is possible that at finite wavevectors they may also decay into the collective mode with lowest energy ( the mode with $k=2$, which has a gap at $\left.\bar{\omega}_{c}\right)$. 


\subsection{Optical properties and experiments}

In this section we discuss the experimental consequences of the results that we have just derived. The density correlation function can be probed by optical absorption and by Raman-scattering experiments.

In the first case, the optical absorption is proportional to the imaginary part of the density correlation function. We predict that there will be absorbtion peaks at a discrete set of frequencies which, for $\vec{Q} \rightarrow 0$ converge to $\omega=k \bar{\omega}_{c}$, where $k$ is an integer number greater than two. Since the spectral weight of these modes vanishes as $\vec{Q} \rightarrow 0$, the associated absorption peaks are, for a strictly translationally invariant system, only observable at non-zero momentum.

In the case of the Raman scattering, the geometry must be such that there is a component of the incident light wavevector in the plane of the sample. The Raman spectrum, $I(\omega)$ jis also proportional to the imaginary part of the density correlation function 20 .

We have seen that in the limit $|\vec{Q}| \ll \ell^{-1}$, where $\ell$ is the magnetic length, most of the weight of $K_{00}(\omega, \vec{Q})$ is in one of the cyclotron modes. The pole in $K_{00}(\omega, \vec{Q})$ for the lowest excitation frequency, $\omega_{k}$ with $k=2$, has a residue which is proportional to $|\vec{Q}|^{4}, i$. e., it is smaller by a factor of $|\vec{Q}|^{2}$ than the residue at the highest weighted mode at the cyclotron frequency.

We have also found that there is a splitting in the cyclotron modes. If the pair potential has a gradient expansion in $|\vec{Q}|, i$. e., short range interaction, the pole at $\omega_{-}$(Eq. 47), has a residue that is smaller by a factor of $|\vec{Q}|^{2(m-3)}$ than the residue of $\omega_{+}$(Eq. 45). The relative Raman intensity, $\frac{I\left(\omega_{+}\right)}{I\left(\omega_{-}\right)}$, is proportional to $\left(\frac{2 B_{\text {eff }}}{\bar{Q}^{2}}\right)^{(m-3)}$ which is a big number within our approximation. If the filling fraction is $\nu=\frac{1}{3}$, both modes have the same $\vec{Q}^{2}$ dependence in their spectral weight, but the relative intensity is $\approx 2.5$ provided that $\tilde{V}(0)=0$. Except for $\nu=\frac{1}{3}$, the splitting between the two modes at the cyclotron frequency satisfies, at leading order in $|\vec{Q}|, \triangle \omega^{2}=\omega_{+}^{2}-\omega_{-}^{2}=\omega_{+}^{2}-\omega_{c}^{2}$, which is proportional to $|\vec{Q}|^{2}$. Up to this order, experimentally one should observe one mode dispersing as $\omega_{+}$(Eq. 15), and the other as $\omega=\omega_{c}$. For $\nu=\frac{1}{3}$ the splitting is also proportional to $|\vec{Q}|^{2}$. In this case one should observe both modes $\omega_{+}$and $\omega_{-}$, Eq. 42), but with different intensities.

If the pair potential has the Coulomb form, the residue of $\omega_{-}$(Eq. 51) is smaller by a factor of $|\vec{Q}|^{2(m-2)}$ than the residue of $\omega_{+}$(Eq. 49), and this is valid for all the values of the filling fraction that we have studied. The splitting between these two modes satisfies, at leading order in $|\vec{Q}|, \triangle \omega^{2}=\omega_{+}^{2}-\omega_{c}^{2}$, 
which is proportional to $|\vec{Q}|$. For $\nu$ different from $\frac{1}{3}$, the relative intensity between the two modes is proportional to $\left(2 B_{\text {eff }} / \vec{Q}^{2}\right)^{(m-3)} M \tilde{V}(\vec{Q})$, which is bigger than 1 within our approximation. For $\nu=\frac{1}{3}$, the relative intensity is proportional to $M \tilde{V}(\vec{Q})$. This factor can be written in terms of the magnetic length and the cyclotron energy as follows $\frac{\tilde{V}(\vec{Q}) / \ell}{\omega_{c}}$. Since our approximation is only valid in the limit $\frac{1}{|\vec{Q}|} \gg \ell$, the numerator satisfies $\tilde{V}(\vec{Q}) / \ell \gg \frac{2 \pi q^{2}}{\ell}$. The second term in this inequality is the Coulomb energy at the magnetic length, which is typically of the same order of magnitude than the cyclotron energy. Therefore, $\frac{\tilde{V}(\vec{Q}) / \ell}{\omega_{c}} \gg \frac{2 \pi q^{2} / \ell}{\omega_{c}} \approx 1$. In other words, the relative intensity for $\nu=\frac{1}{3}$ is also bigger than one.

\subsection{Saturation of the $f$-sum rule}

We show now that the long wavelength form of $K_{00}$, found at this semiclassical level, saturates the $f$-sum rule. This result implies that the non-gaussian corrections do not contribute at very small momentum. In Section 5 we will use this result to show that the absolute value squared wave function of all the (incompressible) liquid states has the Laughlin form at very long distances, in the thermodynamic limit.

The retarded density and current correlation functions of this theory are, by definition

$$
D_{\mu \nu}^{R}(x, y)=-i \theta\left(x_{0}-y_{0}\right) \times<G\left|\left[J_{\mu}(x), J_{\nu}(y)\right]\right| G>
$$

where $J_{\mu}(\mu=0,1,2)$ are the conserved currents of the theory defined by Eq. 13, and $\mid G>$ is the ground state of the system. Using this definition and the commutation relations between the currents, one can derive the $f$ sum rule for the retarded density correlation function $D_{00}^{R}$. In units in which $e=c=\hbar=1$, it states that

$$
\int_{-\infty}^{\infty} \frac{d \omega}{2 \pi} i \omega D_{00}^{R}(\omega, \vec{Q})=\frac{\bar{\rho}}{M} \vec{Q}^{2}
$$

The polarization tensor $K_{00}$ and the density correlation function $D_{00}$ are simply related by $K_{00}=-D_{00}$. From Eq. 37 and 38 we see that the leading order term in $\vec{Q}^{2}$ of the zero-zero component of the electromagnetic response is given by

$$
K_{00}=-\frac{\bar{\rho}}{M} \frac{\vec{Q}^{2}}{\omega^{2}-\omega_{c}^{2}+i \epsilon}
$$


where we have used that $\frac{\bar{\rho}}{B}=\frac{\nu}{2 \pi}$. Thus, we see that the leading order term of $K_{00}$ saturates the $f$-sum rule, Eq. 53 .

It is important to remark that the coefficient of the leading order term of $K_{00}$ can not be renormalized by higher order terms in the gradient expansion, nor in the semiclassical expansion. In the case of the gradient expansion, it is clear that higher order terms have higher order powers of $\vec{Q}^{2}$, and then, do not modify the leading order term. In the case of the corrections to $K_{00}$ originating in higher order terms in the semiclassical expansion, they also come with higher order powers of $\vec{Q}^{2}$. The reason of that is essentially the gauge invariance of the system. This implies that the higher order correlation functions must be transverse in real space, or equivalently they have higher order powers of $\vec{Q}^{2}$ in momentum space. Being higher order terms in the $\vec{Q}^{2}$ expansion they can not change the leading order term.

As we have already mentioned, these results hold for any model Hamiltonian for the two-dimensional electron gas (2DEG) with reasonably local interactions, $i$. e., with pair interactions that obey $\vec{Q}^{2} \tilde{V}(Q) \rightarrow 0$ as $\vec{Q}^{2} \rightarrow 0$.

\subsection{Hall Conductance, Effective Low Energy Action and Quantum Numbers}

We show now that, already within our approximations, this state does exhibit the Fractional Hall Effect with the exact value of the Hall conductance. In order to do so, we will calculate the Hall conductance of the whole system. Since we are only interested in the leading long-distance behavior, it is sufficient to keep only those terms in Eq. 36 which have the smallest number of derivatives, or in momentum space, the smallest number of powers of $\vec{Q}$. Therefore, from Eq. 36 and 37, we see that the leading long distance behavior (i. e., small momentum) of the effective action for the electromagnetic field is governed by the Chern-Simons term. In this limit Eq. 36 turns out to be

$$
S_{\mathrm{eff}}^{\mathrm{em}}\left(\tilde{A}_{\mu}\right) \approx-\frac{i}{2} \int \frac{d^{2} Q d \omega}{(2 \pi)^{3}} \tilde{A}_{\mu}(-\omega,-\vec{Q}) K_{1}(\omega, \vec{Q}) \epsilon_{\mu \nu \lambda} Q^{\lambda} \tilde{A}_{\nu}(\omega, \vec{Q})
$$

where $Q^{0}=\omega$ and $Q^{i}=-Q_{i}$.

To study the Hall response of the system, we will now consider the limit of small $\omega$ and small $\vec{Q}$. We have checked that in this theory the two limits commute

$$
\begin{aligned}
& \lim _{\vec{Q} \rightarrow 0} \lim _{\omega \rightarrow 0} K_{1}(\omega, \vec{Q})=\frac{\Pi_{1}(0,0)}{\theta+\Pi_{1}(0,0)} \\
& \lim _{\omega \rightarrow 0} \lim _{\vec{Q} \rightarrow 0} K_{1}(\omega, \vec{Q})=\frac{\Pi_{1}(0,0)}{\theta+\Pi_{1}(0,0)}
\end{aligned}
$$


This is a consequence of the incompressibility of the ground state. Since $\theta=$ $\frac{1}{2 \pi 2 s}$ and $\Pi_{1}(0,0)=\frac{p}{2 \pi}$

$$
K_{1} \rightarrow \frac{\nu}{2 \pi} \equiv \theta_{\text {eff }}
$$

where $\nu$ is the filling fraction. The electromagnetic current $J_{\mu}$ induced in the system is obtained by differentiating the effective action $S_{\text {eff }}\left(\tilde{A}_{\mu}\right)$ with respect to the electromagnetic vector potential. The current is $J_{\mu}=\frac{\theta_{\text {eff }}}{2} \epsilon_{\mu \nu \lambda} \tilde{F}^{\nu \lambda}$. Thus, if a weak external electric field $\tilde{E}_{j}$ is applied, the induced current is $J_{k}=\theta_{\text {eff }} \epsilon_{l k} \tilde{E}_{l}$. We can then identify the coefficient $\theta_{\text {eff }}$ with the actual Hall conductance of the system $\sigma_{x y}$ and get

$$
\sigma_{x y} \equiv \theta_{\mathrm{eff}}=\frac{\nu}{2 \pi}
$$

which is a fractional multiple of $\frac{e^{2}}{h}$ (in units in which $e=\hbar=1$ ). Thus, the uniform states exhibit a Fractional Quantum Hall effect with the correct value of the Hall conductance.

We can also derive these results by considering an effective action for the low energy excitations. At the gaussian level, the effective action is obtained by keeping only those terms in Eq. 28 which have the smallest number of derivatives and we can then approximate $S_{\text {eff }}$ by

$$
S_{\mathrm{eff}} \approx \frac{\sigma_{x y}^{0}}{4} S_{\mathrm{CS}}\left(\tilde{a}_{\mu}\right)+\frac{\theta}{4} S_{\mathrm{CS}}\left(\tilde{a}_{\mu}-\tilde{A}_{\mu}\right) .
$$

where the induced Hall conductance $\sigma_{x y}^{0}= \pm \frac{p}{2 \pi}$. Although we have obtained this result within the gaussian approximation, it should be stressed that $\sigma_{x y}^{0}$ does not get corrected to any order in perturbation theory. This is so because, at least for a system with a gap and in the thermodynamic limit, $\sigma_{x y}^{0}$ is determined by a topological invariant 28,29, the First Chern Character $\mathcal{C}$. In the problem at hand, we have $\mathcal{C}=p$. Thus, the Hall conductance that we will calculate below is actually exact.

Upon integrating over the statistical vector potentials, we find that the effective action for the electromagnetic fields is

$$
S_{\mathrm{eff}}^{\mathrm{em}}\left(\tilde{A}_{\mu}\right) \approx \frac{\theta_{\mathrm{eff}}}{4} S_{\mathrm{CS}}\left(\tilde{A}_{\mu}\right)
$$

where $\theta_{\text {eff }}$ is given by

$$
\frac{1}{\theta_{\mathrm{eff}}}=\frac{1}{\theta}+\frac{1}{\sigma_{x y}^{0}} .
$$

i. e., the Chern-Simons coupling constants are added "in parallel". 
The values of $\theta$ and $\sigma_{x y}^{0}$ determined above yield the result

$$
\theta_{\text {eff }}=\frac{\nu}{2 \pi}
$$

where $\nu$ is the filling fraction. As before, we obtain the value of the Hall conductance from the electromagnetic current $J_{\mu}$ induced in the system. The result is $\sigma_{x y} \equiv \theta_{\text {eff }}=\frac{\nu}{2 \pi}$. Notice that the coefficient $\sigma_{x y}^{\text {eff }}$ of the effective action represents the integer Hall effect of the bound states and it is different from the true Hall conductance $\sigma_{x y}$.

Finally, we discuss the charge and statistics of the topological excitations, the quasiparticles and quasiholes. We recall that, at the level of the Average Field Approximation, the single particle excitations are fermions, single (charge one) composite fermions. However, the fluctuations of the statistical gauge fields modify this picture in an essential way. For instance, if we want to compute the effective charge of these excitations, we need to find the exact low energy behavior of the composite fermion propagator. Since, even at the level of the AFA, these single particle states have a finite energy gap, the Feynman path integral form of their propagator is dominated by smooth paths $\Gamma$. The fermions couple to both, the statistical and electromagnetic fields as if they had charge one. It was shown in reference 30 that the leading effect of the fluctuations of the gauge fields can be found by computing the expectation value of a Wilson loop operator using the effective action of Eq. 60. More explicitly, we find

$$
\left\langle e^{i \int_{\Gamma} d x_{\mu}\left(a^{\mu}(x)+A^{\mu}(x)\right)}\right\rangle_{\mathrm{eff}}=e^{i \gamma(\Gamma)}\left\langle e^{i \int_{\Gamma} d x_{\mu} \tilde{a}^{\mu}(x)}\right\rangle_{\mathrm{eff}}
$$

Here $\gamma(\Gamma)$ is the Aharonov-Bohm phase factor

$$
\gamma(\Gamma)=\frac{2 \pi}{2 s p \pm 1} \frac{\Phi_{0}(\Gamma)}{\phi_{0}}
$$

where $\Phi_{0}(\Gamma)=B L(\Gamma)^{2}$ is the flux through the area enclosed by $\Gamma$ and $\phi_{0}$ is the flux quantum. On the l.h.s. of this equation $a^{\mu}$ is the complete Chern-Simons gauge field, i.e., the sum of the solution of the SPE plus the fluctuating part $\tilde{a}^{\mu}$. Eq. 65 tells us that the effective charge of this excitation is $q_{\mathrm{eff}}=\frac{e}{2 s p \pm 1}$. The additional phase factor can, in principle, give rise to a fractional spin but it is not possible to determine its value (or even if it exists!) without making contact with the microscopic theory.

The statistics of these excitations can be computed using a similar type of reasoning. Now we need to look at a two-particle propagator and, consequently, we need to compute expectation values of two Wilson loops. In section 2 we gave a prescription for the computation of the expectation values that we need 
here. The effective value of $\theta$ in Eq. 11 now is $\bar{\theta}=\theta+\sigma_{x y}^{0}$. Thus, the statistical angle $\delta$ (measured from fermions) is

$$
\delta=\frac{1}{2 \bar{\theta}}=\pi \frac{2 s}{2 s p \pm 1}
$$

Hence, at long distances and at low energies, the composite fermions are actually fractionally charged anyons. This is the well know result of Arovas, Scrhieffer and Wilczek 31 .

\section{Fermionic Chern-Simons theory for the Fractional Quantum Hall Effect in bilayers and partially polarized systems}

If one allows for the presence of new degrees of freedom, a richer variety of states can be found. The two obvious possibilities that one can consider are systems in which the electronic spin is not frozen by the Zeeman energy, and systems in which two or more layers of $2 \mathrm{DES}$ are coupled together. For instance, the experimentally obseryed $\nu=\frac{5}{2}$ state 32 , has been explained theoretically by Haldane and Rezayi 33 using the fact that the system is not spin-polarized.

Due to continuing advances in material-growth techniques, it has been possible to fabricate high-quality multiple $2 \mathrm{DE}$ layers in close proximity. In these systems the layer index is the new degree of freedom, and the interplay between the intralayer and the interlayer Coulomb interactions gives rise to very interesting physics. In particular, this competition can explain 34 the experimental observation 35 of the destruction or weakening of the IQHE at odd filling fractions. Another interesting case is the one of the $\nu=\frac{1}{2}$ state. In single-layer systems, even though many transport anomalies have been reported there is no evidence of FQHE. On the other hand, this is a well observed 36 FQHE state in double-layer systems.

There are two energy scales that play a very important role in bilayers. One is the potential energy between the electrons in different layers, and the other one is the tunneling amplitude between layers. In the case in which tunneling between the layers may be neglected, the number of particles in each layer is conserved. The system has an effective $U(1) \otimes U(1)$ symmetry reflecting the separate conservation of charge in each layer. Tunneling processes break this symmetry down to a global $U(1)$ invariance. In the next subsection we will describe the $U(1) \otimes U(1)$ fermionic Chern-Simons theory for bilayers which ignores interlayer tunneling effects. In a subsequent section we present an $S U(2) \otimes U(1)$ fermionic Chern-Simons theory. This theory describes the physics of spin as well as tunneling effects in bilayers. 


\subsection{Fermionic Chern-Simons theory for double layer FQHE systems}

The action for a double-layer 2DES in the presence of an external uniform magnetic field $B$ perpendicular to it is given by

$$
\begin{aligned}
\mathcal{S}= & \int d^{3} z \sum_{\alpha}\left\{\psi_{\alpha}^{*}(z)\left[i D_{0}+\mu_{\alpha}\right] \psi_{\alpha}(z)-\frac{1}{2 M}\left|\vec{D} \psi_{\alpha}(z)\right|^{2}\right\} \\
& -\frac{1}{2} \int d^{3} z \int d^{3} z^{\prime} \sum_{\alpha, \beta}\left(\left|\psi_{\alpha}(z)\right|^{2}-\bar{\rho}_{\alpha}\right) V_{\alpha \beta}\left(\left|\vec{z}-\vec{z}^{\prime}\right|\right)\left(\left|\psi_{\beta}\left(z^{\prime}\right)\right|^{2}-\bar{\rho}_{\beta}\right)
\end{aligned}
$$

where the indices $\alpha=1,2$ and $\beta=1,2$ label the layers, $\bar{\rho}_{\alpha}$ is the average particle density in the layer $\alpha, \psi(z)_{\alpha}$ is a second quantized Fermi field, $\mu_{\alpha}$ is the chemical potential and $D_{\mu}$ is the covariant derivative which couples the fermions to the external electromagnetic field $A_{\mu}$. In what follows we will assume that the pair potential has either the Coulomb form, i. e.,

$$
V(|\vec{r}|)_{\alpha \beta}=\frac{q^{2}}{\sqrt{\left.\vec{r}^{2}+\vec{d}^{2}\left(1-\delta_{\alpha \beta}\right)\right)}}
$$

(with $d$ the interlayer separation), or that it represents a short range interaction such that in momentum space it satisfies that $V_{\alpha \beta}(\vec{Q}) \vec{Q}^{2}$ vanishes at zero momentum. This includes the case of ultralocal potentials $(i . e$. , with a range smaller or of the same order as the cyclotron length $\ell$ ), in which case we can set $\tilde{V}(0)=0$, or short range potentials with a range longer than $\ell$ such as a Yukawa interaction.

Using a generalization of the methods of Section 2, it is possible to show 37 that this system is equivalent to a system of interacting electrons coupled to an additional statistical vector potential $a_{\alpha}^{\mu}(\mu=0,1,2)$ whose dynamics is governed by the Chern-Simons action

$$
\mathcal{S}_{\mathrm{cs}}=\sum_{\alpha \beta} \frac{\kappa_{\alpha \beta}}{2} \int d^{3} x \epsilon^{\mu \nu \lambda} a_{\mu}^{\alpha} \partial_{\nu} a_{\lambda}^{\beta}
$$

provided that the CS coupling constant matrix satisfies

$$
\kappa^{\alpha \beta}=\frac{1}{2 \pi\left(4 s_{1} s_{2}-n^{2}\right)}\left(\begin{array}{cc}
2 s_{2} & -n \\
-n & 2 s_{1}
\end{array}\right)
$$

where $s_{1}, s_{2}$ and $n$ are arbitrary integers. In Eq. $69 x_{0}, x_{1}$ and $x_{2}$ represent the time and the space coordinates of the electrons respectively. In the equivalent 
theory the covariant derivative is given by

$$
D_{\mu}^{\alpha}=\partial_{\mu}+i \frac{e}{c} A_{\mu}+i a_{\mu}^{\alpha}
$$

and it couples the fermions to the statistical gauge fields $\left(a_{\mu}^{\alpha}\right)$, and to the external electromagnetic field $\left(A_{\mu}\right)$. Notice that the theory has now a $U(1) \otimes$ $U(1)$ gauge invariance.

The Chern-Simons coupling constant matrix $\kappa^{\alpha \beta}$ of Eq. 70 must be nonsingular. For the case of a singular matrix, it is better to arrange the gauge fields in linear combinations $a_{ \pm}^{\mu}$ which diagonalize the coupling matrix. In general this situation arises only if the matrix elements satisfy $\kappa_{11}=\kappa_{22} \equiv \kappa$ and $\kappa_{12}=\kappa_{21}=\kappa^{\prime}$ and the matrix is singular if $\kappa=\kappa^{\prime}$. In this case the gauge field $a_{-}^{\mu}$ acts like a local constraint on the allowed states demanding that $\rho_{1}(\vec{x}, t)=\rho_{2}(\vec{x}, t)$. In other words, the allowed states consist of pairs of fermions, one from each layer. If we think of the layer index as spin, these states are local spin singlets and the allowed states are spinless bosons of charge 2 (with zero transversal spatial extent). Thus, for a singular coupling matrix, this theory effectively describes a FQHE of a single layer of charge 2 bosons. While this is prefectly correct, it is clear that this is an extreme case and that this approach is uncapable of describing a singlet FQH state not made of local singlets. To describe a state of this sort within a Chern-Simons approach it is required to attach separate fluxes to each species of fermions in a manner consistent to the full $S U(2)$ symmetry of spin. To describe this situation correctly it is necessary to use a non-abelian approach, which we present in Section 4.4 .

There is another limit of physical interest. Clearly, in a bilayer system (or for a single layer with spin) it should also be possible to attach the same flux (using only one gauge field) to the fermions of both layers. This situation is achieved by taking the limit $\kappa-\kappa^{\prime} \rightarrow \infty$ (which kills the field $a_{-}^{\mu}$ ) while holding $\frac{1}{2}\left(\kappa+\kappa^{\prime}\right)$ finite. This is the conventional scheme used to describe partially polarized FQH states 38 .

The Chern-Simons action implies a constraint for the particle density $j_{0}^{\alpha}(\vec{x})$ and the statistical flux $\mathcal{B}^{\alpha}=\epsilon_{i j} \partial_{i} a_{j}^{\alpha}$, given by

$$
j_{0}^{\alpha}(\vec{x})+\kappa_{\alpha \beta} \mathcal{B}_{\beta}(\vec{x})=0
$$

(from now on we assume that repeated indices are contracted).

This relation states that the electrons in plane $\alpha$ coupled to statistical gauge fields with Chern-Simons coupling constant given by Eq. 70, see a statistical flux per particle of $2 \pi 2 s_{\alpha}$ for the particles in their own plane, and a statistical flux per particle of $2 \pi n$ for the particles in the opposite plane. 
(Notice that in units in which $e=c=\hbar=1$, the flux quantum is equal to $2 \pi$ ). If the coefficient of the Chern-Simons term is chosen with the above prescription, all the physical amplitudes calculated in this theory are identical to the amplitudes calculated in the standard theory, in which the Chern-Simons field is absent. Of course, this is true provided that the dynamics of the statistical gauge fields is fully taken into account exactly.

Here we take into account the dynamics of the Chern-Simons gauge fields in a semiclassical expansion, which is a sequence of well controlled approximations. In practice, we will only consider the leading and next-to-leading order in the semiclassical approximation.

Using the constraint enforced by the Chern-Simons action, the interaction term of the action Eq. 67 becomes

$$
\mathcal{S}_{\text {int }}=-\frac{1}{2} \int d^{3} z \int d^{3} z^{\prime}\left(\kappa^{\alpha \delta} \mathcal{B}_{\delta}(z)-\bar{\rho}_{\alpha}\right) V_{\alpha \beta}\left(\left|\vec{z}-\vec{z}^{\prime}\right|\right)\left(\kappa^{\beta \gamma} \mathcal{B}_{\gamma}\left(z^{\prime}\right)-\bar{\rho}_{\beta}\right)
$$

The quantum partition function for this problem is, at zero temperature

$$
\mathcal{Z}\left[A_{\mu}\right]=\int \mathcal{D} \psi^{*} \mathcal{D} \psi \mathcal{D} a_{\mu}^{\alpha} \exp \left(i S\left(\psi^{*}, \psi, a_{\mu}^{\alpha}, A_{\mu}\right)\right)
$$

Since the action is quadratic in the fermions, they can be integrated out. The effective action $\left(\mathcal{S}_{\text {eff }}\right)$ is given by (in units in which $e=c=\hbar=1$ )

$$
\mathcal{S}_{\text {eff }}=-i \sum_{\alpha} \operatorname{tr} \ln \left\{i D_{0}^{\alpha}+\mu_{\alpha}+\frac{1}{2 M}\left(\vec{D}^{\alpha}\right)^{2}\right\}+\mathcal{S}_{\mathrm{cs}}\left(a_{\mu}^{\alpha}-\tilde{A}_{\mu}^{\alpha}\right)+\mathcal{S}_{\text {eff }}^{\text {int }}\left(a_{\mu}^{\alpha}-\tilde{A}_{\mu}^{\alpha}\right)
$$

where

$$
\begin{aligned}
\mathcal{S}_{\text {eff }}^{\text {int }}\left(a_{\mu}^{\alpha}-\tilde{A}_{\mu}^{\alpha}\right)=\quad & -\frac{1}{2} \int d^{3} z \int d^{3} z^{\prime}\left(\kappa^{\alpha \delta}\left(\mathcal{B}_{\delta}(z)-\tilde{B}_{\delta}(z)\right)-\bar{\rho}_{\alpha}\right) \\
& V_{\alpha \beta}\left(\left|\vec{z}-\vec{z}^{\prime}\right|\right)\left(\kappa^{\beta \gamma}\left(\mathcal{B}_{\gamma}\left(z^{\prime}\right)-\tilde{B}_{\gamma}\left(z^{\prime}\right)\right)-\bar{\rho}_{\beta}\right)
\end{aligned}
$$

Here we have written the external electromagnetic field as a sum of two terms, one representing the uniform magnetic field $B$, and a small fluctuating term $\tilde{A}_{\mu}^{\alpha}$ whose average vanishes everywhere. The latter will be used to probe the electromagnetic response of the system. Notice that we have used the invariance of the measure $\mathcal{D} a_{\mu}^{\alpha}$ with respects to shifts, to move $\tilde{A}_{\mu}^{\alpha}$ out of the covariant derivatives and into the Chern-Simons and the interaction terms of the effective action (Eq. 75).

The path integral $\mathcal{Z}$ can be approximated by expanding its degrees of freedom in powers of the fluctuations, around stationary configurations of $\mathcal{S}_{\text {eff }}$. 
This requirement yields the SPE or classical equations of motion.

$$
\begin{aligned}
<j_{0}^{\alpha}(z)>_{F}= & -\kappa_{\alpha \beta}\left[<\mathcal{B}^{\beta}(z)>-\tilde{B}^{\beta}(z)>\right] \\
<j_{k}^{\alpha}(z)>_{F}= & -\kappa_{\alpha \beta} \epsilon_{k l}\left[<\mathcal{E}_{l}^{\beta}(z)>-\tilde{E}_{l}^{\beta}(z)>\right] \\
& +\epsilon_{k l} \partial_{l}^{z} \int d^{3} z^{\prime} \kappa^{\alpha \epsilon} V_{\epsilon \delta}\left(z, z^{\prime}\right)\left[-\kappa^{\delta \gamma}<\mathcal{B}^{\gamma}-\tilde{B}^{\gamma}>\left(z^{\prime}\right)-\bar{\rho}^{\delta}\right]
\end{aligned}
$$

Just as in the case of the single layer problem, these equations have many possible solutions. The uniform solutions of Eq. 77 represent the FQH fluid states of the bilayer system at the mean field level. In this state, the electrons in the layer $\alpha$ see a total flux $B_{\text {eff }}^{\alpha}$, equal to the external magnetic flux partially screened by the average Chern-Simons flux, i. e. $B_{\text {eff }}^{\alpha}=B+\left\langle\mathcal{B}^{\alpha}\right\rangle=B-$ $\left(\kappa^{-1}\right)^{\alpha \beta} \bar{\rho}_{\beta}$. It is easy to see that the uniform saddle-point state has a gap only if the effective field $B_{\text {eff }}^{\alpha}$ is such that the fermions in layer $\alpha$ fill exactly an integer number $p_{\alpha}$ of the effective Landau levels, $i$. e., those defined by $B_{\text {eff }}^{\alpha}$. In other words, the AFA to this theory yields a state with an energy gap if the filling fractions of each layer satisfy

$$
\begin{aligned}
& \nu_{1}=\frac{1}{ \pm \frac{1}{p_{1}}+2 s_{1}+n \frac{N_{2}}{N_{1}}} \\
& \nu_{2}=\frac{1}{ \pm \frac{1}{p_{2}}+2 s_{2}+n \frac{N_{1}}{N_{2}}}
\end{aligned}
$$

where $N_{1}$ and $N_{2}$ are the number of particles in layers 1 and 2 respectively. The sign in front of $p_{1}$ and $p_{2}$ indicates if the effective field is parallel or antiparallel to the external magnetic field.

Using the fact that $\nu=\nu_{1}+\nu_{2}$, and that the number of flux quanta enclosed by each plane is the same, the filling fractions can be written as follows

$$
\begin{aligned}
& \nu_{1}=\frac{n-\left( \pm \frac{1}{p_{2}}+2 s_{2}\right)}{n^{2}-\left( \pm \frac{1}{p_{1}}+2 s_{1}\right)\left( \pm \frac{1}{p_{2}}+2 s_{2}\right)} \\
& \nu_{2}=\frac{n-\left( \pm \frac{1}{p_{1}}+2 s_{1}\right)}{n^{2}-\left( \pm \frac{1}{p_{1}}+2 s_{1}\right)\left( \pm \frac{1}{p_{2}}+2 s_{2}\right)}
\end{aligned}
$$

where $p_{1}, p_{2}, s_{1}, s_{2}$ and $n$ are arbitrary integers. For the special case in which the two layers have the same occupancy, $N_{1}=N_{2}$ and $\nu_{1}=\nu_{2}=\frac{\nu}{2}$, the allowed fractions are

$$
\nu(p, n, s)=\frac{2 p}{(n+2 s) p+1}
$$


where $p$ is an arbitrary (positive or negative) integer.

The effective magnetic field can be written in terms of the external magnetic field as

$$
B_{\text {eff }}^{\alpha}=B \frac{\nu^{\alpha}}{p^{\alpha}}
$$

For general values of these integers, the states whose filling fractions are given by Eq. 79 have a gap at the mean field level of this approximation. This ensures that the perturbative expansion is meaningful. If there is no gap for the excitations of the mean field ground state, the perturbative expansion breaks down. The breakdown is signalled by infrared divergencies at low temperatures. Such is the case for the compressible or "Fermi Liquid" states.

It is clear from Eq. 79 that in the bilayer systems, there are many possible choices of the numbers $p_{1}, p_{2}, s_{1}, s_{2}$ and $n, i$. e., many different states, which have the same filling fractions. These states are physically different since, as we will see, the quantum numbers of their excitation spectra are different. This is an example of the concept of topological order that has been formulated by X. G. Wen 39 . More recently, Haldane 40 has proposed an algebraic criterion to determine what states are topologically stable. We will see that all the solutions with an energy gap for all excitations are topologically stable in the sense of Haldane.

As a result of the presence of many solutions, the phase diagram for bilayers is much richer than for spin polarized electrons in a single layer. Which particular solution is actually realized is determined by the microscopic dynamics of a particular system and it requires a detailed calculation of the ground state energy beyond the Average Field Approximation. Experiments on spin polarized 2DEG bilayers 35.36 at filling fractions $\nu=1,1 / 2$ have shown a nontrivial phase diagram with at least two phases: a compressible phase in the regime in which the 2DEG's are well separated and an incompressible thase when they are closer by. However, as it was emphasized by Wen and Zee 11 , the incompressible states at these two filling fractions actually have quite different properties. We will see now that the phase diagram can be quite complex.

Let us consider some cases of special interest, in particular with $N_{1}=N_{2}$. For example, at level one of the hierarchy, we choose $p_{1}=p_{2}=1,2 s_{1}+1=m_{1}$ and $2 s_{2}+1=m_{2}$, and we obtain the so called $\left(m_{1}, m_{2}, n\right)$ states $41,42.43$ whose filling fractions are $\nu=\frac{m_{1}+m_{2}-2 n}{m_{1} m_{2}-n^{2}}$. In particular, for $m_{1}=m_{2}=3$ and $n=1$, this is the $(3,3,1)$ state, whose filling fraction is $\nu=\frac{1}{2}$. For $n_{1}=p_{2}=1$, and $2 s_{1}+1=2 s_{1}+1=n=m$, we obtain the $(m, m, m)$ states 41 . 4 whose filling fractions are $\nu=\frac{1}{m}$.

The states $\left(m_{1}, m_{2}, n\right)$ are the equivalent of the Laughlin states for bilayers and as such should be regarded as primary states. Hence, for each $\left(m_{1}, m_{2}, n\right)$ 
primary state there is a hierarchy of states labelled by the index $p$. For example, the primary state $(1,1,1)$, defined by $p=1, s=0$ and $n=1$, has the hierarchy $\nu_{+}(p, 1,0)=\frac{2 p}{p+1}=1, \frac{4}{3}, \frac{3}{2}, \frac{8}{5}, \ldots 2$. The first three states in this series have already been seen in experiments 45 . Similarly, the primary state $(3,3,1)$ (which has $p=1, n=s=1)$, has the hierarchy $\nu_{+}(p, 1,1)=\frac{2 p}{3 p+1}=\frac{1}{2}, \frac{4}{7}, \frac{3}{5}, \ldots, \frac{2}{3}$. Recall that $\nu_{ \pm}(p, n, s)$ is the total filling fraction of the bilayer and that the individual filling fractions of each layer are $\nu / 2$.

We can also consider the limit $p_{1}=p_{2} \equiv p \rightarrow \infty$. In this case, if $s_{1}=s_{2}=$ $s$, the state with filling fraction $\nu=\frac{1}{m}$ will be obtained for all the values of $n$ and $s$ such that $n+2 s=2 m$. Since $p \rightarrow \infty$, the effective field vanishes, and we find the analogous of the compressible states for the single layer problem discussed by Halperin et al 16 . All of these states are degenerate with the $\nu=\frac{1}{m}$ states mentioned above.

Clearly, it is always possible to construct a large family of, in principle, distinct states which have the same filling fraction. In particular, new states are generated by transferring intra-plane flux-particle attachment, determined by $s_{1}$ and $s_{2}$, to inter-plane flux attachment, determined by $n$. Since the interplane attached fluxes are negative in these states, the particles of one plane see the particles of the other plane as if they were holes. Thus, in these states, there is an effective attractive force between particles on different planes. Therefore the wave functions, instead of having zeros of higher order (which represent repulsion), should have a larger weight when particles from different planes are closer to each other (in the sense of their coordinates projected on the $x y$ plane). Asymptotically, these wave functions appear to have "poles" in the interlayer coordinates. One could think of this state as a condensate of interlayer bound states. Such states should become preferable as the interlayer coupling constant becomes large.

\subsection{Spectrum of bilayer FQH states}

Following the same steps as in Section 3.1, we can integrate out the gaussian fluctuations of the statistical gauge fields $\tilde{a}_{\mu}^{\alpha}$, and obtain the effective action for the electromagnetic fluctuations $\tilde{A}_{\mu}^{\alpha}$

$$
\mathcal{S}_{\text {eff }}^{\mathrm{em}}\left(\tilde{A}_{\mu}^{\alpha}\right)=\frac{1}{2} \int d^{3} x \int d^{3} y \tilde{A}_{\mu}^{\alpha}(x) K_{\alpha \beta}^{\mu \nu}(x, y) \tilde{A}_{\nu}^{\beta}(y)
$$

Here $K_{\alpha \beta}^{\mu \nu}$ is the electromagnetic polarization tensor, which measures the linear response of the system to a weak electromagnetic perturbation. As for the single-layer systems, we can use this effective action to calculate the full electromagnetic response functions at the gaussian level. 
In this Section we discuss the spectrum of excitations for two different states, the $(m, m, n)$ and the $(m, m, m)$ states. The derivation of these results can be found in reference 37 .

We first discuss the case of the $(m, m, n)$ states. For simplicity, we study the collective modes for the state $(3,3,1)$. All the other states can be studied by straightforward application of the same methods 37 .

In this case the total filling fraction is $\nu=\frac{1}{2}$, being $\nu_{1}=\nu_{2}=\frac{1}{4}$. The effective cyclotron frequencies and magnetic fields are $\omega_{\text {eff }}^{1}=\omega_{\text {eff }}^{2}=\bar{\omega}=\frac{\omega_{c}}{4}$ and $B_{\text {eff }}^{1}=B_{\text {eff }}^{2}=\bar{B}=\frac{B}{4}$.

We find that there is a family of collective modes whose zero-momentum gap is $k \bar{\omega}$, where $k$ is an integer number different from 1. At mean field level, there are two modes for each integer multiple of $\bar{\omega}$. After including the gaussian fluctuations we find that there are no modes with a zero momentum gap at $\bar{\omega}$. One of them has been "pushed up" to the cyclotron frequency and the other up to $2 \bar{\omega}($ at $\vec{Q}=0)$. Therefore, at these multiples of $\bar{\omega}$ there are three degenerated modes for $\vec{Q}=0$. For $\vec{Q} \neq 0$, the degeneracy is lifted and these three modes have different dispersion curves.

At $2 \bar{\omega}$ we find that there are two modes with residue $\vec{Q}^{2}$, and one with residue $\vec{Q}^{4}$. The former are

$$
\omega_{ \pm}(\vec{Q})=\left[(2 \bar{\omega})^{2}+\left(\frac{\vec{Q}^{2}}{2 B_{\mathrm{eff}}}\right)^{\frac{1}{2}} \bar{\omega}^{2} \alpha_{ \pm}\right]^{\frac{1}{2}}
$$

where

$$
\alpha_{ \pm}=\frac{3 M}{2 \pi}\left(v_{11}-v_{12}\right) \pm \sqrt{\left(\frac{3 M}{2 \pi}\right)^{2}\left(v_{11}-v_{12}\right)^{2}+16}
$$

Here $v_{\alpha \beta}$ are the zeroth order coefficients of the Fourier transform of the interparticle pair potential for short range interactions. For Coulomb interactions $v_{11}=\frac{q^{2}}{\epsilon}$ and $v_{12}=\frac{q^{2}}{\epsilon} e^{-|\vec{Q}| d} \approx \frac{q^{2}}{\epsilon}$ if $|\vec{Q}| d \ll 1$, therefore $\alpha_{ \pm}= \pm 4$ in this limit.

The residues in $K_{00}^{\alpha \beta}$ corresponding to these poles are

$$
\operatorname{Res}\left(K_{00}^{\alpha \beta}, \omega_{ \pm}(\vec{Q})\right)=-\vec{Q}^{2} \omega_{c} \frac{\nu}{8 \pi}\left(\begin{array}{cc}
1 & -1 \\
-1 & 1
\end{array}\right)\left(1+\frac{16}{\alpha_{ \pm}^{2}}\right)^{-1}
$$

It is clear from the form of these residues, that these excitations are out of phase modes, because they only couple the "out of phase" density (i.e., they couple $\rho^{-}$with itself).

The other mode at $2 \bar{\omega}$ is

$$
\omega_{0}(\vec{Q})=\left[(2 \bar{\omega})^{2}-6\left(\frac{\vec{Q}^{2}}{2 B_{\mathrm{eff}}}\right) \bar{\omega}^{2}\right]^{\frac{1}{2}}
$$


and its residue is proportional to $\vec{Q}^{4}\left(\begin{array}{ll}1 & 1 \\ 1 & 1\end{array}\right)$. Thus, this is an in phase mode since it only couples $\rho^{+}$with itself.

There are three modes whose zero momentum frequency is the cyclotron frequency. The only mode with residue $\vec{Q}^{2}$ has energy

$$
\omega(\vec{Q})=\left[\omega_{c}^{2}+\left(2+\frac{M\left(v_{11}+v_{12}\right)}{\pi}\right) 3\left(\frac{\vec{Q}^{2}}{2 B_{\mathrm{eff}}}\right) \bar{\omega}^{2}\right]^{\frac{1}{2}}
$$

and residue

$$
\operatorname{Res}\left(K_{00}, \omega(\vec{Q})\right)=-\vec{Q}^{2} \omega_{c} \frac{\nu}{8 \pi}\left(\begin{array}{ll}
1 & 1 \\
1 & 1
\end{array}\right),
$$

As in the case of single layers, except for the lowest branch of collective modes and the cyclotron mode at $\vec{Q}=0$, all the other modes become damped due to processes induced by the non-quadratic interactions among the collective modes.

We now discuss the collective modes of the $(m, m, m)$ states. In this case the total filling fraction is $\nu=\frac{1}{m}$, being $\nu_{1}=\nu_{2}=\frac{1}{2 m}$. The effective cyclotron frequencies and magnetic fields are $\omega_{\text {eff }}^{1}=\omega_{\text {eff }}^{2} \equiv \bar{\omega}=\frac{\omega_{c}}{2 m}$ and $B_{\text {eff }}^{1}=B_{\text {eff }}^{2} \equiv B_{\text {eff }}=\frac{B}{2 m}$.

We find again a family of collective modes whose zero-momentum gap is $k \bar{\omega}$, where $k$ is an integer number different from 1. At mean field level, there are two modes for each integer multiple of $\bar{\omega}$. After including the gaussian fluctuations we find that there are no modes with a zero momentum gap at $\bar{\omega}$. One of them has been "pushed up" to the cyclotron frequency. Therefore, at $\omega_{c}$ there are three degenerate modes for $\vec{Q}=0$. For $\vec{Q} \neq 0$, the degeneracy is lifted and these three modes have different dispersion curves. The other mode at $\bar{\omega}$ has been "pulled down" to zero frequency at $\vec{Q}=0, i$. e., it has become a gapless mode.

We will distinguish between the cases $m=1$ and $m \neq 1$.

a) Case $m=1$

The gapless mode is

$$
\omega(\vec{Q})=v_{s}|\vec{Q}|
$$

where

$$
v_{s}^{2}=\left[1+\frac{M}{2 \pi}\left(v_{11}-v_{12}\right)\right] \frac{\omega_{c} \nu}{2 M}
$$

where $v_{\alpha \beta}$ are the zeroth order coefficient of the Fourier transform of the interparticle pair potential for short range interactions. For Coulomb interactions $v_{11}(\vec{Q})=\frac{q^{2}}{\epsilon}$ and $v_{12}(\vec{Q})=\frac{q^{2}}{\epsilon} e^{-|\vec{Q}| d} \approx \frac{q^{2}}{\epsilon}$ if $|\vec{Q}| d \ll 1$. Therefore, $\left(v_{11}-v_{12}\right)=0$ for Coulomb interactions (in the limit $|\vec{Q}| d \ll 1$ ). 
The residue in $K_{00}^{\alpha \beta}$ corresponding to this pole is

$$
\operatorname{Res}\left(K_{00}, \omega(\vec{Q})\right)=-\vec{Q}^{2} \omega_{c} \frac{\nu}{8 \pi}\left(\begin{array}{cc}
1 & -1 \\
-1 & 1
\end{array}\right)
$$

Therefore, this is an out of phase mode.

At $\omega_{c}=2 \bar{\omega}$ we find that there are two (in phase) modes with residue $\vec{Q}^{2}$

$$
\omega_{ \pm}(\vec{Q})=\left[\omega_{c}^{2}+\left(\frac{\vec{Q}^{2}}{2 B_{\mathrm{eff}}}\right)^{\frac{1}{2}} \bar{\omega}^{2} \alpha_{ \pm}\right]^{\frac{1}{2}}
$$

where

$$
\alpha_{ \pm}=\frac{M}{2 \pi}\left(v_{11}+v_{12}\right) \pm \sqrt{\left(\frac{M}{2 \pi}\right)^{2}\left(v_{11}+v_{12}\right)^{2}+16}
$$

For Coulomb interactions $v_{11}+v_{12}=2 \frac{q^{2}}{\epsilon}$ if $|\vec{Q}| d \ll 1$, therefore this term is higher order in $\vec{Q}$ and it should be neglected, $i$. e., $\alpha_{ \pm}=4$.

The residues in $K_{00}^{\alpha \beta}$ corresponding to these poles are

$$
\operatorname{Res}\left(K_{00}, \omega_{ \pm}(\vec{Q})\right)=-\vec{Q}^{2} \omega_{c} \frac{\nu}{8 \pi}\left(\begin{array}{ll}
1 & 1 \\
1 & 1
\end{array}\right)\left(1+\frac{16}{\alpha_{ \pm}^{2}}\right)^{-1}
$$

therefore these are in phase modes. The other mode at $\omega_{c}$ is

$$
\omega_{0}(\vec{Q})=\left[\omega_{c}^{2}-2\left(\frac{\vec{Q}^{2}}{2 B_{\text {eff }}}\right) \bar{\omega}^{2}\right]^{\frac{1}{2}}
$$

and its residue is proportional to $\vec{Q}^{4}\left(\begin{array}{cc}1 & -1 \\ -1 & 1\end{array}\right)$ (out of phase mode).

b) Case $m \neq 1$

The gapless mode is an out of phase mode with the same form as for $m=1$ (Eq. 89) and with the same residue (Eq. 91). $\vec{Q}^{2}$

At $\omega_{c}=2 m \bar{\omega}$ we find that there is only one in phase mode with residue

$$
\omega(\vec{Q})=\left[\omega_{c}^{2}+\left(\frac{2 m-1}{m-1}+\frac{M}{2 \pi}\left(v_{11}+v_{12}\right)\right) 2 m\left(\frac{\vec{Q}^{2}}{2 B_{\mathrm{eff}}}\right) \bar{\omega}^{2}\right]^{\frac{1}{2}}
$$

The residue in $K_{00}^{\alpha \beta}$ corresponding to this pole is

$$
\operatorname{Res}\left(K_{00}, \omega(\vec{Q})\right)=-\vec{Q}^{2} \omega_{c} \frac{\nu}{8 \pi}\left(\begin{array}{ll}
1 & 1 \\
1 & 1
\end{array}\right)
$$

The modes with zero momentum frequency $\omega=k \bar{\omega}$ for $k \geq 3$ have been calculated in reference 37 .

All the considerations about the validity of this spectrum of collective excitations beyond the semiclassical approximation that we discussed in previous sections are of course valid in this case as well. 


\subsection{Effective Low Energy Action, Hall Conductance and Quantum Numbers}

We will derive now the effective action for the low energy degrees of freedom of the bilayer system. Using the same line of argument of Section 3.5, we keep only the leading terms in a gradient expansion and find

$$
\begin{aligned}
S_{\mathrm{eff}}\left(\tilde{a}_{\mu}^{\alpha}, \tilde{A}_{\mu}^{\alpha}\right) & \approx \frac{\bar{\kappa}^{\alpha \beta}}{2} \int d^{3} x \tilde{a}_{\mu}^{\alpha} \epsilon_{\mu \nu \lambda} \partial^{\lambda} \tilde{a}_{\nu}^{\beta}-\frac{\kappa^{\alpha \beta}}{2} \int d^{3} x \tilde{a}_{\mu}^{\alpha} \epsilon_{\mu \nu \lambda} \partial^{\lambda} \tilde{A}_{\nu}^{\beta} \\
& -\frac{\kappa^{\alpha \beta}}{2} \int d^{3} x \tilde{A}_{\mu}^{\alpha} \epsilon_{\mu \nu \lambda} \partial^{\lambda} \tilde{a}_{\nu}^{\beta}+\frac{\kappa^{\alpha \beta}}{2} \int d^{3} x \tilde{A}_{\mu}^{\alpha} \epsilon_{\mu \nu \lambda} \partial^{\lambda} \tilde{A}_{\nu}^{\beta}
\end{aligned}
$$

where we have set $\bar{\kappa}^{\alpha \beta}=\frac{p_{\alpha}}{2 \pi} \delta^{\alpha \beta}+\kappa^{\alpha \beta}$.

The effective action of Eq. 98 is very similar to the effective action of Wen and Zee's Matrix classification of Abelian quantum Hall states 4 . It differs from it in two respects: in Eq. 98 there is an induced Chern-Simons term for the background electromagnetic fields, and the coefficients are not quite the same as in reference 46 . However, as we will see below, Eq. 98 leads to the correct value of the Hall conductance and of the fractional charge and statistics of the quasiparticles. Thus, they describe the same physics.

The next step is to integrate the statistical gauge fields to obtain the effective action for the electromagnetic field. In particular, we will need to compute the inverse of the matrix $\bar{\kappa}^{\alpha \beta}$. This inverse only exists if $\Delta=\left[\left(\frac{1}{p_{1}}+\right.\right.$ $\left.\left.2 s_{1}\right)\left(\frac{1}{p_{2}}+2 s_{2}\right)-n^{2}\right] \neq 0$. Therefore we must consider two cases, the one in which $\Delta \neq 0$ and the one in which $\Delta=0$.

Case $\Delta \neq 0$

Upon integrating over the statistical gauge fields in Eq. 98, the effective action for the electromagnetic field results

$$
S_{\mathrm{eff}}^{\mathrm{em}}\left(\tilde{A}_{\mu}^{\alpha}\right) \approx \frac{\kappa_{\mathrm{eff}}^{\alpha \gamma}}{2} \int d^{3} x \tilde{A}_{\mu}^{\alpha} \epsilon_{\mu \nu \lambda} \partial^{\lambda} \tilde{A}_{\nu}^{\beta}
$$

where the effective quantum Hall conductance matrix $\kappa_{\mathrm{eff}}^{\alpha \beta}$

$$
\kappa_{\mathrm{eff}}^{\alpha \beta}=\kappa^{\alpha \gamma}\left[\delta^{\gamma \beta}-\left(\bar{\kappa}^{-1}\right)^{\gamma \delta} \kappa^{\delta \beta}\right]
$$

is given by

$$
\kappa_{\mathrm{eff}}^{\alpha \beta}=\frac{1}{2 \pi\left[\left(\frac{1}{p_{1}}+2 s_{1}\right)\left(\frac{1}{p_{2}}+2 s_{2}\right)-n^{2}\right]}\left(\begin{array}{cc}
\left(\frac{1}{p_{2}}+2 s_{2}\right) & -n \\
-n & \left(\frac{1}{p_{1}}+2 s_{1}\right)
\end{array}\right)
$$


The matrix $\kappa_{\text {eff }}^{\alpha \beta}$ gives the intralayer and translayer conductances of the bilayer system. The translayer conductances characterize the Coulomb drag effects in bilayers.

In particular, let us consider the case in which both layers are coupled to the same electromagnetic field. Then $\tilde{A}_{\mu}^{1}=\tilde{A}_{\mu}^{2}=\tilde{A}_{\mu}$, and the coefficient in the effective action results

$$
\kappa_{\mathrm{eff}} \equiv \sum_{\alpha \beta} \kappa_{\mathrm{eff}}^{\alpha \beta}=\frac{1}{2 \pi} \frac{2 n-\left(\frac{1}{p_{1}}+2 s_{1}\right)-\left(\frac{1}{p_{2}}+2 s_{2}\right)}{n^{2}-\left(\frac{1}{p_{1}}+2 s_{1}\right)\left(\frac{1}{p_{2}}+2 s_{2}\right)}=\frac{\nu}{2 \pi}
$$

Following the same arguments as in Section 3.5 we find that the electromagnetic current induced in the system is $J_{\mu}=\frac{\kappa_{\text {eff }}}{2} \epsilon_{\mu \nu \lambda} \tilde{F}^{\nu \lambda}$. Thus, if a weak external electric field $\tilde{E}_{j}$ is applied, the induced current is $J_{k}=\kappa_{\text {eff }} \epsilon_{l k} \tilde{E}_{l}$. Therefore the coefficient $\kappa_{\text {eff }}$ is the actual Hall conductance of the system.

$$
\sigma_{x y} \equiv \kappa_{\mathrm{eff}}=\frac{\nu}{2 \pi}
$$

which is a fractional multiple of $\frac{e^{2}}{h}$ (in units in which $e=\hbar=1$ ). Thus, the uniform states exhibit a Fractional Quantum Hall effect with the correct value of the Hall conductance.

The effective action of Eq. 98 also determines the quantum numbers (i. e., charge and statistics) of the vortices (topological excitations) supported by this FQH state. These quantum numbers were calculated in reference 37 and we will not give an explicit derivation here. The charge of the excitations was found to be given by

$$
q_{\mathrm{eff}}^{\alpha}=\frac{\nu^{\alpha}}{p^{\alpha}}
$$

In particular, for the $(m, m, n)$ states, the effective charge in both layers is the same and is given by $q_{\mathrm{eff}}=\frac{1}{n+m}$.

On the other hand, the statistics of the excitations on the same layer is

$$
\delta_{\alpha}=\pi\left(1+\frac{\left(p_{\beta}+\frac{2 s_{\alpha}}{4 s_{1} s_{2}-n^{2}}\right)}{\left(p_{1}+\frac{2 s_{2}}{4 s_{1} s_{2}-n^{2}}\right)\left(p_{2}+\frac{2 s_{1}}{4 s_{1} s_{2}-n^{2}}\right)-\frac{n^{2}}{\left(4 s_{1} s_{2}-n^{2}\right)^{2}}}\right)
$$

where if $\alpha=1$, then $\beta=2$ and viceversa. In particular, for the $(m, m, n)$ states, the statistics is $\delta=-\frac{m}{\left(m^{2}-n^{2}\right)}$, independent of the layer. The relative statistics (i. e., for excitations on different layers) $\delta_{12}$ is given by

$$
\delta_{12}=\pi \frac{\frac{n}{4 s_{1} s_{2}-n^{2}}}{\left(p_{1}+\frac{2 s_{2}}{4 s_{1} s_{2}-n^{2}}\right)\left(p_{2}+\frac{2 s_{1}}{4 s_{1} s_{2}-n^{2}}\right)-\frac{n^{2}}{\left(4 s_{1} s_{2}-n^{2}\right)^{2}}}
$$


In particular, for the $(m, m, n)$ states, the relative statistics results $\delta=\frac{n}{\left(m^{2}-n^{2}\right)}$.

It is worth to discuss these results in the context of Haldanes's topological stability criterion 40 . After some (tedious) algebra it is straightforward to show that Haldanes's topological stability is equivalent to the requirement that the denominators in Eq. 105 be non-zero. For the special case of the $(m, m, n)$ states, this holds if $m \neq n$. We will see next that this requirement fails if there exists one branch of collective excitations with a gapless mode, such as in the $(m, m, m)$ states.

Case $\Delta=0$

It can be shown that when $\bar{\kappa}^{\alpha \beta}$ is not invertible, $i . e$., it has a zero eigenvalue, the corresponding linear combination of the gauge fields become massless. In other words, the in phase gauge field $\tilde{a}_{\mu}^{+}=\tilde{a}_{\mu}^{1}+\tilde{a}_{\mu}^{2}$ has a finite gap which couples to the electromagnetic field $\tilde{A}_{\mu}$, while the out of phase gauge field $\tilde{a}_{\mu}^{-}=\tilde{a}_{\mu}^{1}-\tilde{a}_{\mu}^{2}$ is gapless.

We will study in particular the case of the $(m, m, m)$ states which satisfy the condition $\Delta=0$. For these states

$$
\bar{\kappa}=\frac{-m}{2 \pi(1-2 m)}\left(\begin{array}{cc}
1 & 1 \\
1 & 1
\end{array}\right)
$$

which is clearly non invertible.

We can write the effective action for the fluctuations of the Chern-Simons gauge fields (Eq. 98) in the basis defined by $\tilde{a}_{\mu}^{ \pm}=\tilde{a}_{\mu}^{1} \pm \tilde{a}_{\mu}^{2}$.

$$
\begin{aligned}
S_{\mathrm{eff}}\left(\tilde{a}_{\mu}^{\alpha}, \tilde{A}_{\mu}^{\alpha}\right) & \approx-\frac{1}{2} \int d^{3} x \frac{m}{2 \pi(1-2 m)} \tilde{a}_{\mu}^{+} \epsilon_{\mu \nu \lambda} \partial^{\lambda} \tilde{a}_{\nu}^{+} \\
& -\frac{1}{2} \int d^{3} x \frac{1}{4 \pi(1-2 m)}\left(-\tilde{a}_{\mu}^{+} \epsilon_{\mu \nu \lambda} \partial^{\lambda} \tilde{A}_{\nu}^{+}+(2 m-1) \tilde{a}_{\mu}^{-} \epsilon_{\mu \nu \lambda} \partial^{\lambda} \tilde{A}_{\nu}^{-}\right) \\
& -\frac{1}{2} \int d^{3} x \frac{1}{4 \pi(1-2 m)}\left(-\tilde{A}_{\mu}^{+} \epsilon_{\mu \nu \lambda} \partial^{\lambda} \tilde{a}_{\nu}^{+}+(2 m-1) \tilde{A}_{\mu}^{-} \epsilon_{\mu \nu \lambda} \partial^{\lambda} \tilde{a}_{\nu}^{-}\right) \\
& +\frac{1}{2} \int d^{3} x \frac{1}{4 \pi(1-2 m)}\left(-\tilde{A}_{\mu}^{+} \epsilon_{\mu \nu \lambda} \partial^{\lambda} \tilde{A}_{\nu}^{+}+(2 m-1) \tilde{A}_{\mu}^{-} \epsilon_{\mu \nu \lambda} \partial^{\lambda} \tilde{A}_{\nu}^{-}\right)
\end{aligned}
$$

The gauge field $\tilde{a}_{\nu}^{-}$appears as a Lagrange multiplier in this action. The integration over it states that the current $\epsilon_{\mu \nu \lambda} \partial^{\lambda} \tilde{A}_{\nu}^{-}$vanishes. This is trivially valid if the electromagnetic field is the same for both layers, because $\tilde{A}_{\nu}^{-}=0$.

The integration over $\tilde{a}_{\nu_{\tilde{A}}}^{+}$gives the effective action for the field $\tilde{A}_{\nu}^{+}$. If we consider the case in which $\tilde{A}_{\mu}^{1}=\tilde{A}_{\mu}^{2}=\tilde{A}_{\mu}$, the result is

$$
S_{\text {eff }}^{\mathrm{em}}\left(\tilde{A}_{\mu}\right)=\frac{1}{2 \pi m} \int d^{3} x \tilde{A}_{\mu} \epsilon_{\mu \nu \lambda} \partial^{\lambda} \tilde{A}_{\nu}
$$


Following the same steps as in the previous case, the Hall conductance results $\sigma_{x y}=\frac{1}{2 \pi m}=\frac{\nu}{2 \pi}$ which is the correct value for the $(m, m, m)$ states.

The existence of a gapless branch in the spectrum of collective excitations has important consequences for the spectrum of topological excitations $i$. e., quasiparticles and quasiholes. It was shown in reference 37 that the gapless collective excitations mediate an effective confining gauge force between topological excitations which carry the quantum numbers of the out-of-phase (or inter-layer) mode. The result is that the only surviving excitations are bound states which are neutral with respect to the inter-layer gauge group but carry charge $1 / m$ and fractional statistics $\pi / m$ (measured from fermions). These states have a finite energy gap. For the particular case of the state $(1,1,1)$, these bound states are bosons of charge 1 and finite gap. This gapped bound state has the same quantum numbers as the skyrmion excitation of the $(1,1,1)$ discussed by K. Yang et. al., who view the $(1,1,1)$ state as a quantum ferromagnet 4 . S. L. Sondhi and collaborators had predicted earlier that a partially polarized $\nu=1 \mathrm{QH}$ state should have skyrmion states 38 . Our results indicate that all of the $(m, m, m)$ states should have skyrmion excitations but, in general, they have fractional charge $1 / m$ and fractional statistics $\pi / m$. Hence, the bilayer state $(3,3,3)$, which occurs at $\nu=1 / 3$ (1/6 on each layer), has skyrmions of charge $1 / 3$ and statistics $\pi / 3$.

The reduction of the number of topological excitations for the case $m=n$ illustrates, in a physically transparent way, the meaning of Haldane's criterion for topological stability. Indeed, Haldane's criterion predicts that the $(m, m, m)$ states are not topologically stable. This of course does not mean that the state itself is unstable or unphysical. Rather, it means that if one or more branches of collective excitations become gapless, the quasiparticles which couple to them are not present in the spectrum and the corresponding quantum numbers are not allowed.

\subsection{Spin Singlet States and SU(2) Symmetry}

In this Section we discuss the application of Chern-Simons methods to a 2DEG with an $S U(2)$ symmetry. This symmetry may he exact or may be broken by explicit terms in the Hamiltonian. In reference 48 a non-abelian Chern-Simons approach was used to construct a theory of the spin singlet $(m+1, m+1, m)$ Halperin states. Here, we present a generalization of the approach of reference 48 which will yield the $S U(2)$ hierarchies. A related, and completely equivalent approach was developed independently by Frohlich, Kerler, and Marchetti 49 .

In the approach of Balatsky and Fradkin 4 (BF), instead of the twocomponent abelian Chern-Simons theory that we use in this Chapter, a non- 
abelian Chern-Simons gauge field is introduced. The advantage of the BF approach is that the $S U(2)$ invariance is manifest and it is not the consequence of a subtle dynamical mechanism. The disadvantage of the BF approach is that the non-abelian Chern-Simons theory is substantially more sophisticated and technically more demanding than the abelian theory used before. In the $\mathrm{BF}$ approach, the electron is viewed as a composite object which is made of a particle that carries the charge (the holon) and another particle that carries the spin (the spinon). This arbitrary separation gives rise to the existence of an abelian gauge symmetry (called $R V B$ by $\mathrm{BF}$ ). The requirement of gauge invariance forces the holons and spinons to be glued together in bound states, the electrons. In the BF approach, the need of a non-abelian gauge field is a consequence of the assignment of fractional (semion) statistics to both holons and spinons. In this way, $S U(2)$ fluxes are attached to a set of charge neutral, spin- $\frac{1}{2}$, fermions, which become the spinons. The $S U(2)$ symmetry only admits Bose, Fermi or semionic statistics. The holons, instead, are represented by fermions attached to $U(1)$ fluxes. The $U(1)$ and $S U(2)$ Chern-Simons coupling constants must be chosen in such a way that the holons and spinons are semions. Within this approach, the FQHE of the spin-singlet states is the FQHE of the semions. The spin structure just sits on top of the FQHE.

There is another, and more obvious, way to attach fluxes to particles while keeping the $S U(2)$ spin symmetry untouched. Belkhir and Jain 50 proposed a spin singlet wave function for the state with filling fraction $\nu=\frac{1}{2}$, based on a composite fermion picture. In their construction, they attach the same number of pairs of flux quanta to both up and down spins. These approaches are represented in the $U(1) \otimes U(1)$ theory of the preceeding sections by demanding that both up and down electrons see that same flux at all times. This means to choose a Chern-Simons matrix $\kappa^{\alpha \beta}$ which is proportional to the identity, i. e., $s_{1}=s_{2}$ and $n=0$. It is easy to use this approach to get the $S U(2)$ limit of the Jain states, but with arbitrary polarization. However, it is not a very efficient approach to get all of the spin singlet states. The approach of BF deals with this states more directly. Alternatively, it is possible to attach flux to the charge degrees of freedom, leaving spin untouched. This construction, but in the bosonic Chern-Simons language, was used by D. H. Lee and C Kane 51 and subsequently applied by Sondhi, Karlhede, Kivelson and Rezayi 33 in their theory of skyrmion states in polarized FQHE states. This approach does not describe the spin singlet states. In what follows, we follow the BF approach.

More concretely, we introduce a holon field $\phi$ and a spinon field $\chi_{\alpha}(\alpha=\uparrow$ $, \downarrow)$ and represent the electron field operator $\psi_{\alpha}$ as $\psi_{\alpha}(x)=\phi(x) \chi_{\alpha}(x)$. BF showed that, for an $S U(2)$ invariant system, the system defined by the action of Eq. 67 is equivalent to the following theory of (interacting) spinons and 
holons

$$
\mathcal{S}=\mathcal{S}_{\text {charge }}+\mathcal{S}_{\text {spin }}+\mathcal{S}_{\text {interaction }}
$$

where the action for the charge degrees of freedom is

$$
\begin{aligned}
\mathcal{S}_{\text {charge }} & =\int d^{3} x\left(\phi^{\dagger}(x)\left(i D_{0}^{c}+\mu\right) \phi(x)+\frac{1}{2 M} \phi^{\dagger}(x) \vec{D}_{c}^{2} \phi(x)\right) \\
& +\int d^{3} x \frac{\theta}{2} \epsilon_{\mu \nu \lambda} a^{\mu}(x) \partial^{\nu} a^{\lambda}(x)
\end{aligned}
$$

while the action for spin is

$$
\begin{aligned}
\mathcal{S}_{\text {spin }} & =\int d^{3} x\left(\chi_{\alpha}^{\dagger}(x) i D_{0}^{s} \chi_{\alpha}(x)+\frac{1}{2 M} \chi_{\alpha}^{\dagger}(x) \vec{D}_{s}^{2} \chi_{\alpha}(x)\right) \\
& -\int d^{3} x \frac{k}{4 \pi} \epsilon_{\mu \nu \lambda} \operatorname{tr}\left(b^{\mu}(x) \partial^{\nu} b^{\lambda}(x)+\frac{2}{3} b^{\mu}(x) b^{\nu}(x) b^{\lambda}(x)\right)
\end{aligned}
$$

and an (instantaneous) pair interaction term

$$
\mathcal{S}_{\text {interaction }}=-\int d^{3} x \int d^{3} x^{\prime} \frac{1}{2}(\rho(x)-\bar{\rho}) V\left(x-x^{\prime}\right)\left(\rho\left(x^{\prime}\right)-\bar{\rho}\right)
$$

where $\rho(x)$ is the charge density

$$
\rho(x)=\psi_{\alpha}^{\dagger}(x) \psi_{\alpha}(x) \equiv \phi^{\dagger}(x) \phi(x)
$$

(see below). In Eq. 111, $a_{\mu}$ is the statistical vector potential which turns the holons into semions. This condition requires that the $U(1)$ Chern-Simons coupling constant $\theta$ be restricted to the values

$$
\frac{1}{\theta}=-\frac{2 \pi}{m}+2 \pi 2 s
$$

where semion statistics requires that $m= \pm 2$ and $s$ is an arbitrary integer. Likewise, in Eq. $112 b_{\mu}$ is the $S U(2)$ non-abelian statistical gauge field which takes values on the $S U(2)$ algebra. Hence, we can expand the field in the form $b_{\mu}(x)=b_{\mu}^{a}(x) \tau^{a}$, where $\tau^{a}(a=1,2,3)$ are the three generators of $S U(2)$ in the spinor representation, $i$. e., the set of $2 \times 2$ Pauli matrices. The $S U(2)$ Chern-Simons coupling constant $k$, the level of the Chern-Simons theory is, for our system, equal to $k= \pm 1$. Hence, we have a ("trivial") level one ChernSimons theory. All the representations of a level one Chern-Simons theory are known to be abelian and to correspond to abelian fractional statistics of fermions, bosons or semions 0 . The only ambiguity left is the sign of $k$ which is 
the chirality (or handedness) of the semion. There is a similar sign ambiguity in the coupling constant $\theta$ in Eq. 115. Different choices of these signs lead to different statistics for the bound states of holons and spinons. The requirement that the bound state be an electron, which is a fermion, leads to the condition $\operatorname{sign}(k)=\operatorname{sign}(m)$.

The $U(1)$ and $S U(2)$ charge and spin covariant derivatives are

$$
\begin{aligned}
& D_{\mu}^{c}=\partial_{\mu}-i\left(A_{\mu}+a_{\mu}+c_{\mu}\right) \\
& D_{\mu}^{s}=I \partial_{\mu}-i\left(b_{\mu}-I c_{\mu}\right)
\end{aligned}
$$

where $A_{\mu}$ is the external electromagnetic field and $I$ is the $2 \times 2$ identity matrix. The gauge field $c_{\mu}$ is the $U(1)$ " $R V B$ " gauge field which glues spins and charges together. The covariant derivatives have been chosen in such a way that holons and spinons have opposite charge with respect to $c_{\mu}$. Hence, the strong fluctuations of this field binds holons and spinons into states which are locally singlets under the "RVB" gauge transformations, $i$. e., electrons. In fact, all the gauge field $c_{\mu}$ does is to enforce the constraint that the 3 -current of the holons equals the 3 -current of the spinons, as an operator statement in the physical Hilbert space. This is seen clearly from the equation of motion generated by $c_{\mu}$

$$
J^{\mathrm{RVB}}(x)=\frac{\delta \mathcal{S}}{\delta c_{0}(x)}=0 \Longrightarrow \phi^{\dagger}(x) \phi(x)-\sum_{\sigma=\uparrow, \downarrow} \chi_{\sigma}^{\dagger}(x) \chi_{\sigma}(x)=0
$$

Thus $N_{\mathrm{e}}$, the number of charges, and $N_{\uparrow}$ and $N_{\downarrow}$, the number of up and down spins, must obey the obvious relation $N_{\mathrm{e}}=N_{\uparrow}+N_{\downarrow}$. Also, Eq. 117 tells us that the local particle density operator $\rho(x)$ can be identified with the holon charge density operator, i. e., $\rho(x)=\phi^{\dagger}(x) \phi(x)$.

In the way the theory has been set up, it may appear that this theory may apply only if $S U(2)$ is an exact symmetry. However, this is not the case. Let us consider for instance the effects of an $S U(2)$ symmetry breaking field of the form of a Zeeman term in the action

$$
\mathcal{S}_{\text {Zeeman }}=\int d^{3} x \vec{B} \cdot \psi_{\alpha}^{\dagger}(x) \vec{\tau}_{\alpha \beta} \psi_{\beta}(x)
$$

Using the definition $\psi_{\alpha}(x)=\phi(x) \chi_{\alpha}(x)$ and the constraint Eq. 117, we can write the Zeeman term in the equivalent form

$$
\mathcal{S}_{\text {Zeeman }}=\int d^{3} x \vec{B} \cdot \chi_{\alpha}^{\dagger}(x) \vec{\tau}_{\alpha \beta} \chi_{\beta}(x)
$$


Hence, the Zeeman operators of Eq. 118 and Eq. 119 have exactly the same form. This is an exact identity in the restricted Hilbert space of states satisfying the constraint of Eq. 117. Likewise, spin-spin pair interaction exchange terms can be written exactly in the same way. Clearly, these rules also apply to any term involving spins even if the symmetry is not respected. Thus, a bilayer 2DEG is a system of fermions with a layer label which can be regarded as a "spin" index, even though the $S U(2)$ symmetry is actually broken. The interlayer tunneling term can be regarded as Zeeman term. Similarly, the matrix pair interaction of the electrons in the bilayer system can be represented, in the $S U(2)$ formulation, in terms of a suitable combination of the $S U(2)$ invariant charge pair interaction and an Ising interaction term.

We will not go into the details of the full non-abelian theory. Instead, we will use this theory to determine the allowed fractions for $S U(2)$ invariant states. Thus, we will just consider the AFA equations for this theory. There are two sets of AFA equations, one for the charge sector and one for the spin sector. The AFA equations for the charge sector are just the AFA equations for a charged interacting semion liquid with $N_{\mathrm{e}}$ particles in an uniform magnetic field with $N_{\phi}$ flux quanta, $i$. e., a FQHE of semions. A simple application of the methods of Section 4.1 yields the constraint for the charge density operator

$$
\frac{\delta \mathcal{S}}{\delta a_{0}(x)}=0 \Longrightarrow j_{0}(x)=-\theta \mathcal{B}_{c}(x)
$$

where $j_{0}(x)$ is the charge density in the liquid state, $i . e ., \bar{\rho}$, and $\mathcal{B}_{c}(x)$ is the $U(1)$ statistical charge flux. This equation, when specialized on fluid states, yields the allowed fractions. Similar considerations for the $S U(2)$ gauge field $b_{\mu}^{a}$ (with $a=1,2,3$ ) yield a constraint for the local spin density operator

$$
\frac{\delta \mathcal{S}}{\delta b_{\mu}(x)^{a}}=0 \Longrightarrow j_{0}^{a}(x)=-\psi_{\sigma}^{\dagger} \tau^{a} \psi_{\sigma}=\frac{k}{2 \pi} \mathcal{B}_{s}^{a}
$$

where $\mathcal{B}_{s}^{a}$ is the $S U(2)$ spin flux.

For fluid states, we generalize the Average Field Approximation and replace these exact local operator identities by translationally invariant averages. This replacement may be problematic in the case of the $R V B$ gauge field since its only mission is to enforce exactly the constraint Eq. 117. At the level of the wave functions for the allowed ground states, this constraint simply means that every coordinate for a charge degree of freedom has to coincide with the coordinate of a spin degree of freedom.

Thus, we seek fluid states with $N_{e}$ particles, $N_{\phi}$ flux quanta and filling fraction $\nu=N_{e} / N_{\phi}$. We wish to determine the filling fractions and spin for 
which the ground state is a fluid. In addition to the usual AFA equation for the charge, that will give the allowed fractions, we will now get conditions for the spin and polarization of the allowed states.

In the charge sector, we have a FQHE of spinless semions. In this case, the AFA consists of a system of $N_{e}$ spinless fermions filling up effective Landau levels, exactly as in the case of spin polarized electrons (and anyons!). The same line of argument that was used to derive the allowed fractions in Section 4.1 now tells us that, from Eq. 120, the effective number of fluxes is

$$
\bar{N}_{\phi}=N_{\phi}-\frac{N_{e}}{2 \pi \theta}
$$

and, hence, the allowed fractions, $\nu^{ \pm}$, for the $S U(2)$ fluid states satisfy

$$
\frac{1}{\nu^{ \pm}}= \pm \frac{1}{p}-\frac{1}{2 \pi \theta}
$$

where $p$ is a positive integer and the + sign corresponds to a "particle-like" FQHE (i. e., the effective flux parallel to the external flux) while the - sign holds for a "hole-like" FQHE (i. e., the effective flux anti-parallel to the external flux) . By using the allowed values of $\theta$ we find that the allowed fractions for the $S U(2)$ fluid states are of the form

$$
\nu^{ \pm}(p, s ; m)=\frac{m p}{(2 s m-1) p+m} \equiv \frac{2 p}{ \pm 2+(4 s-\operatorname{sign}(m)) p}
$$

where we have specialized for the case of interest, $m=2 \operatorname{sign}(m)$.

The hierarchy of FQHE states of Eq. 124 is a generalization of the states found by $\mathrm{BF}$, which are obtained by setting $p=+1$ and $m=+2, i$.e., $\nu^{+}(1, s ;-2)=\frac{2}{4 s+1}=2, \frac{2}{5}, \frac{2}{9}, \ldots$, which coincide with the Halperin spin singlet states. The state with $\nu=\frac{1}{2}$ is also part of the hierarchy of Eq. 124, where it is realized as the state with $p=+2(-2)$ for $m=+2(-2)$ and $s=1$. Numerical studies show that, for Coulomb-like interactions, this state is not favored and that the $(3,3,1)$ state is an accurate representation of the ground state. Notice that this hierarchy includes a state at filling fraction $\nu=\frac{5}{2}$. This state is found as the $p=-10$ ("hole-like"), $m=-2$ and $s=0$ member of the $S U(2)$ hierarchy, or as the $p_{1}=p_{2}=-5, s_{1}=s_{2}=s$ and $n+2 s=1$ member of the $U(1) \otimes U(1)$ hierarchy.

In contrast with the states found in Section 4.1 using the abelian theory for bilayers, the states of the $S U(2)$ theory at each filling fraction, are arranged in irreducible representations (multiplets) of $S U(2)$. The spin and polarization of the states is determined by Eq. 121. The only subtlety here is that, since 
the components of the total spin do not commute with each other, one can only determine the total spin and total projection. For a system without a boundary, the choice of total spin $S$ and total projection along an arbitrary polarization axis, say $S_{z}$, are constants of motion which are invariant under local $S U(2)$ gauge transformations (but, of course, change under global $S U(2)$ rotations). There are two generic situations of physical interest: (a) spin singlet states (or with microscopic total spin $S / N_{e} \approx O\left(1 / N_{e}\right)$ ) and (b) states with macroscopic spin, $S \approx N_{e}$, i. e., ferromagnetic states. The total z-component of the spin polarization $M=\frac{1}{2}\left(N_{\uparrow}-N_{\downarrow}\right)$ obeys

$$
2 M=N_{\uparrow}-N_{\downarrow}=\frac{k}{2 \pi}\left\langle\mathcal{B}_{3}^{s}\right\rangle L^{2}
$$

where $L^{2}$ is the area. It is clear that it is possible to construct all multiplets with spin $|S| \leq \frac{N_{e}}{2}$. In the thermodynamic limit, the spin singlet states have $S=0$ and, hence, $N_{\uparrow}=N_{\downarrow}=N_{e} / 2$. In contrast, the ferromagnetic states have, with an appropriate choice of the quantization axis, a non-vanishing extensive value of $M$ and, hence, a non-zero value of $\mathcal{B}_{3}^{s}$.

The wave functions for the spin sector of the spin singlet states have to be determined from the states of a level one $S U(2)$ Chern-Simons gauge theory with $N_{e}$ sources in the fundamental representation. It was shown by Witten 8 that these wave functions are correlation functions of conformal blocks of a conformal field theory in two Euclidean dimensions, the $S U(2)$ leyel one Wess-Zumino-Witten model. This fact was used by Read and Moore 22 and by Balatsky and Fradkin 48 to show that, the wave function of the spin singlet FQHE has a factor which is precisely this conformal block correlation function. It was also noticed 48 that this factor coincides with the Kalmeyer-Laughlin wave function 33 for a Spin Liquid.

The states with macroscopic spin have a somewhat different physics. The existence of a non-zero average field should make a mean field approach more sound. The spin sector of this mean field theory has $N_{\uparrow, \downarrow}=\frac{1}{2} N_{e} \pm M$ spin up and spin down spinons each feeling an effective uniform magnetic field of $\pm \frac{2 \pi}{k} M$. Because of the $S U(2)$ invariance, there is no Zeeman term and only the orbital degrees of freedom see this spin-dependent external field. It is easy to see that the highest weight ferromagnetic state with maximal spin is obtained by filling up the lowest Landau level of the up spins while leaving the down spin sector empty.

The charge and spin sectors are not decoupled from each other. Firstly, the constraint of Eq. 117 sets the local charge density to be the same as the local spin density. The wave functions of the allowed states have to satisfy this local property. Secondly, if the system is $S U(2)$ invariant, all of the states 
in a given $S U(2)$ multiplet must have the same filling fraction. Since the fully polarized states have to span all of the Jain states for a single-layer system, we must conclude that the $S U(2)$ states which are not in a main Jain hierarchy cannot achieve the maximum polarization. In other terms, there is an upper bound for the spin polarization and, hence, for the total spin itself. This argument indicates that the filling fraction $\nu$ and the spin $S$ of the state cannot be completely independent from each other for the allowed states. In other words, there should exist a set of selection rules which determines the possible combinations of total spin and filling fraction. Similarly, it should be possible to construct a unified theory of all the FHQE states with $S U(2)$ symmetries, instead of the apparently separate descriptions of the spin singlet and the fully polarizable states that we discuss here.

We conclude this Section with a comparison of the states that are obtained by this $S U(2)$-symmetric approach and the $U(1) \otimes U(1)$ theory. A direct inspection of the allowed fractions, Eq. 79 and Eq. 124, for the $U(1) \otimes U(1)$ and $S U(2)$ theories respectively, shows that they do not yield the same allowed fractions. For instance, the "Fermi Liquid" (compressible) states, with the same occupancy of the two layers, allowed by Eq. 79 have filling fractions $\frac{2}{r}=2,1, \frac{2}{3}, \frac{1}{2}, \frac{2}{5}, \frac{1}{3}, \frac{2}{7}, \frac{1}{4}, \frac{2}{9}, \ldots(r=1,2, \ldots)$. In contrast, the allowed $S U(2)$ "Fermi Liquid" (compressible) states are $\frac{2}{4 s \pm 1}=2, \frac{2}{5}, \frac{2}{3}, \frac{2}{9}, \frac{2}{7}, \ldots$.. Clearly, the fractions $\frac{1}{k}$ (with $k=1,2,3, \ldots$ ) cannot be realized as $S U(2)$ compressible states. Among the states which appear in both hierarchies, we find an incompressible spin unpolarized state at filling fraction $\frac{4}{11}$. It is worth noting that there is experimental evidence of an incompressible state at $\frac{4}{11}$. It is not possible to construct a fully polarized Jain state with this filling fraction for single-layers although it may be constructed as a hierarchical state. It is strange that this is the only observed fraction for which a hierarchical construction is needed. It is believed that the experimentally observed state is polarized. We have checked that all of the states in levels 1 and 2 in the $S U(2)$ hierarchy span the entire level $1 U(1) \otimes U(1)$ states. Similarly, the level 4 $S U(2)$ states span the level $2 U(1) \otimes U(1)$ states, and the levels 3 and $6 S U(2)$ states span the level $3 U(1) \otimes U(1)$ states. However, a large number of incompressible $U(1) \otimes U(1)$ states cannot be realized as $S U(2)$ states. In a way, this may not be surprising since the $U(1) \otimes U(1)$ symmetric theory may only generate $S U(2)$ as a dynamical symmetry. Nevertheless, it is still surprising since in this discussion the form of the interaction terms has not entered and, hence, the symmetries of the Hamiltonian have not had a chance to play any role yet. However, more surprising is the fact that not all of the $S U(2)$ states can be realized as $U(1) \otimes U(1)$ states. It is easy to check, for instance, that the allowed $S U(2)$ state with $\nu=\frac{10}{7}$ and $S_{z}=0$ has no counterpart in the 
$U(1) \otimes U(1)$ states (unless the condition of $S_{z}=0$ is relaxed and polarized states are also considered).

\section{Ground state wave functions}

In this section we will make the connection between the fermion Chern-Simons field theory and the many-body wave-function approach. We will give a description in terms of the bilayer system since it already includes the single layer system.

The first step that we need in order to make this connection explicit is to find the exact asymptotic form of the density-density correlation functions. We need to show first that the long wavelength form of $K_{00}^{\alpha \beta}$, found at this semiclassical level, saturates the $f$-sum rule. This result implies that the nongaussian corrections do not contribute at very small momentum. We will use this result to show that the absolute value squared of the ground state wave function of this state has the Halperin 42 form at very long distances, in the thermodynamic limit.

The $f$-sum rule states that the retarded density-density correlation function $D_{00}^{\alpha \beta}$ satisfies

$$
\int_{-\infty}^{\infty} \frac{d \omega}{2 \pi} i \omega D_{00}^{\alpha \beta}(\omega, \vec{Q})=\frac{\bar{\rho}^{\alpha}}{M} \vec{Q}^{2} \delta^{\alpha \beta}
$$

This equation implies the conservation of the current in each layer separately. It is easy to show that, in the basis of the total and relative currents, $i$. e., in the $\bar{\rho}_{ \pm}=\bar{\rho}^{1} \pm \bar{\rho}^{2}$ basis, Eq. 126 states the conservation of $\bar{\rho}_{+}$and $\bar{\rho}_{-}$independently. On the other hand, from the general definition of the polarization tensor $K_{00}^{\alpha \beta}$, it follows that (see for instance reference 30 )

$$
K_{00}^{\alpha \beta}(x, y)=-D_{00}^{\alpha \beta}(x, y)
$$

Thus, Eq. 126 also holds if we replace $D_{\mu \nu}^{\alpha \beta}$ by $K_{00}^{\alpha \beta}$ (except for an extra minus sign).

\subsection{Ground state wave function for $(m, m, n)$ states}

We have found that for an $(m, m, n)$ state, the leading order term in $\vec{Q}^{2}$ of the zero-zero component of the electromagnetic response is given by

$$
\begin{aligned}
K_{00}^{\alpha \beta}=\quad & -\frac{\bar{\rho}}{4 M} \frac{\vec{Q}^{2}}{\omega^{2}-\omega_{c}^{2}+i \epsilon}\left(\begin{array}{ll}
1 & 1 \\
1 & 1
\end{array}\right) \\
& -\frac{\bar{\rho}}{4 M} \frac{\vec{Q}^{2}}{\omega^{2}-((m-n) \bar{\omega})^{2}+i \epsilon}\left(\begin{array}{cc}
1 & -1 \\
-1 & 1
\end{array}\right)
\end{aligned}
$$


Notice that the poles whose residues in $K_{00}^{\alpha \beta}$ are proportional to $\vec{Q}^{2}$, have zero momentum frequency given by $(m+n) \bar{\omega}=\omega_{c}$ and $(m-n) \bar{\omega}$. It is straightforward to check that Eq. 128 saturates the $f$-sum rule. Therefore, the fermionic Chern-Simons approach gives the correct leading order form for the density correlation function, consistent with the $f$-sum rule, at the semiclassical level of the approximation.

It is important to emphasize that the coefficient of the leading order term of $K_{00}^{\alpha \beta}$ can not be renormalized by higher order terms in the gradient expansion, nor in the semiclassical expansion. In the case of the gradient expansion, it is clear that higher order terms have higher order powers of $\vec{Q}^{2}$, and then, do not modify the leading order term. In the case of the corrections to $K_{00}^{\alpha \beta}$ originating in higher order terms in the semiclassical expansion, they also come with higher order powers of $\vec{Q}^{2}$. The reason for that is the gauge invariance of the system which implies that higher order correlation functions must be transverse in real space or, equivalently, have higher order powers of $\vec{Q}^{2}$ in momentum space. Terms in this expansion higher than $\vec{Q}^{2}$ cannot change the leading order term.

We will now follow the methods of references 22,54 to write the ground state wave function in the density representation. We begin by recalling that the absolute value squared of the ground state wave function in the density representation $\left|\Psi_{0}[\rho]\right|^{2}$ is given by 5

$$
\begin{aligned}
& \left|\Psi_{0}\left[\rho_{1}, \rho_{2}\right]\right|^{2}= \\
& \qquad \mathcal{D} A_{0}^{\alpha} e^{-i \int d^{2} x A_{0}^{\alpha}(\vec{x}) \rho^{\alpha}(\vec{x})} \lim _{A_{0}^{\alpha}(x) \rightarrow A_{0}^{\alpha}(\vec{x}) \delta\left(x_{0}\right)}\left\langle 0\left|T e^{i \int d^{3} x A_{0}^{\alpha}(x) \hat{j}_{0}^{\alpha}(x)}\right| 0\right\rangle
\end{aligned}
$$

where $\hat{j}_{0}^{\alpha}(x) \equiv \hat{\rho}^{\alpha}(x)$.

The operators in this expression are Heisenberg operators of the system in the absence of sources. The vacuum expectation value in the integrand of Eq. 129 can be calculated from the generating functional of density correlation functions, $\mathcal{Z}\left[\tilde{A}_{\mu}^{\alpha}\right]$, defined by

$$
\lim _{A_{0}^{\alpha}(x) \rightarrow A_{0}^{\alpha}(\vec{x}) \delta\left(x_{0}\right)} \mathcal{Z}\left[A_{0}^{\alpha}, \vec{A}^{\alpha}=0\right]=\int \mathcal{D} \psi^{*} \mathcal{D} \psi \mathcal{D} a_{\mu}^{\alpha} e^{i S\left(\psi^{*}, \psi, a_{\mu}^{\alpha}, A_{\mu}^{\alpha}\right)}
$$

Hence, $\left|\Psi_{0}\left[\rho_{1}, \rho_{2}\right]\right|^{2}$ is determined by the generating functional of equal-time density correlation functions.

The path integral on the r. h. s. of Eq. 130 can be written in terms of the effective action $S_{\text {eff }}\left(A_{\mu}^{\alpha}\right)$ for the external electromagnetic field. We have seen that, in the thermodynamic limit, and for weak fields, the effective 
action admits the expansion given by Eq. 32 . Since we need only the density correlation functions, it suffice to know the zero-zero component of $K_{\mu \nu}^{\alpha \beta}$. In momentum space, and in the small $\vec{Q}^{2}$ limit, $K_{00}^{\alpha \beta}$ is given by Eq. 128 . We can see that the dominant term in $K_{00}^{\alpha \beta}$ is of order $1 / B$. Higher order terms in the gradient expansion will contribute with higher powers of $1 / B$. The same observation applies for all the corrections to $K_{00}^{\alpha \beta}$ originating in higher order terms in the semiclassical expansion. Here the thermodynamic limit is crucial since we are only taking into account fluctuations with wavelengths short compared with the linear size of the system. The higher order terms, which vanish like powers of $\vec{Q}^{2} / B$, can only be neglected for an infinite system.

Using the results of Sections 4.1 and 4.2 , Eq. 130 becomes

$$
\lim _{A_{0}^{\alpha}(x) \rightarrow A_{0}^{\alpha}(\vec{x}) \delta\left(x_{0}\right)} \mathcal{Z}\left[A_{0}^{\alpha}, \vec{A}^{\alpha}=0\right]=e^{\frac{i}{2} \int d^{2} x d^{2} y A_{0}^{\alpha}(\vec{x})\left(\lim _{x_{0} \rightarrow y_{0}} K_{00}^{\alpha \beta}(x, y)\right) A_{0}^{\beta}(\vec{y})}
$$

or, by Fourier transforming the exponent, we get

$$
\lim _{A_{0}^{\alpha}(x) \rightarrow A_{0}^{\alpha}(\vec{x}) \delta\left(x_{0}\right)} \mathcal{Z}\left[A_{0}^{\alpha}, \vec{A}^{\alpha}=0\right]=e^{\frac{i}{2} \int \frac{d^{2} Q}{(2 \pi)^{2}} A_{0}^{\alpha}(\vec{Q})\left(\int_{-\infty}^{\infty} \frac{d \omega}{2 \pi} K_{00}^{\alpha \beta}(\omega, \vec{Q})\right) A_{0}^{\beta}(-\vec{Q})}
$$

The terms dropped in the exponent of Eq. 131 and Eq. 132 represent equaltime density correlation functions with more than two densities. These terms give rise to three-body corrections terms ( and higher) to the wave function that modify the Jastrow form. These higher order terms, as we noted above, are of order higher than $\vec{Q}^{2}$ and can be neglected.

Replacing the expression for $K_{00}^{\alpha \beta}(\omega, \vec{Q})$ given by Eq. 128 into Eq. 132 and integrating out $A_{0}^{\alpha}$, we obtain the following form for the absolute value squared of the wave function

$$
\begin{aligned}
\left|\Psi\left(\vec{x}_{1}, \ldots, \vec{x}_{N_{1}}, \vec{y}_{1}, \ldots, \vec{x}_{N_{2}}\right)\right|^{2}= & \\
& \prod_{i<j=1}^{N_{1}}\left|\vec{x}_{i}-\vec{x}_{j}\right|^{2 m} \prod_{i<j=1}^{N_{2}}\left|\vec{y}_{i}-\vec{y}_{j}\right|^{2 m} \prod_{i=1}^{N_{1}} \prod_{j=1}^{N_{2}}\left|\vec{x}_{i}-\vec{y}_{j}\right|^{2 n} \\
& \times \exp \left\{-\frac{B}{2}\left(\sum_{i=1}^{N_{1}}\left|\vec{x}_{i}\right|^{2}+\sum_{i=1}^{N_{2}}\left|\vec{y}_{i}\right|^{2}\right)\right\}
\end{aligned}
$$

where the coordinates $\vec{x}_{i}$ are in plane $1, \vec{y}_{i}$ are in plane 2 , and $N_{1}=N_{2}=\frac{N}{2}$ for this state. Here we have used that the eigenvalues of the local density operator, in a Hilbert space with $N_{\alpha}$ particles, are $\rho^{\alpha}(\vec{x})=\sum_{i=1}^{N_{\alpha}} \delta\left(\vec{x}-\vec{x}_{i}\right)-\bar{\rho}^{\alpha}$. 
The wave function of Eq. 133 is the absolute value squared of the Halperin wave function 42 . Numerical calculations have established 56 that this wave function accurately describes the ground state wave function for the $(3,3,1)$ state for $d=1.5 \ell_{c}$, where $\ell_{c}$ is the cyclotron length. We have shown that Eq. 133 gives the exact form of the ground state wave function at long distances and in the thermodynamic limit. Since the leading order term of $K_{00}^{\alpha \beta}$ saturates the $f$-sum rule, higher order corrections in the expansion cannot modify this result. Arguments similar to the line of reasoning that led to Eq 133 can be used to derive an analogous expression for a single layer system 54 .

\subsection{Ground state wave function for $(m, m, m)$ states}

For these states, the leading order term in $\vec{Q}^{2}$ of the zero-zero component of the electromagnetic response is, according to Eq. 91 and 94 or 97

$$
\begin{aligned}
K_{00}^{\alpha \beta}=\quad & -\frac{\bar{\rho}}{4 M} \frac{\vec{Q}^{2}}{\omega^{2}-\omega_{c}^{2}+i \epsilon}\left(\begin{array}{rr}
1 & 1 \\
1 & 1
\end{array}\right) \\
& -\frac{\bar{\rho}}{4 M} \frac{\vec{Q}^{2}}{\omega^{2}-v^{2} \vec{Q}^{2}+i \epsilon}\left(\begin{array}{rr}
1 & -1 \\
-1 & 1
\end{array}\right)
\end{aligned}
$$

Following the same steps we can prove that the leading order term of $K_{00}^{\alpha \beta}$ saturates the $f$-sum rule, Eq. 126. All the remarks on the exactness of this result are also valid in this case. Substituting Eq. 134 into the expression for the absolute value squared of the ground state wave function (Eq. 132) we obtain

$$
\begin{aligned}
& \left|\Psi\left(\vec{x}_{1}, \ldots, \vec{x}_{N_{1}}, \vec{y}_{1}, \ldots, \vec{x}_{N_{2}}\right)\right|^{2}= \\
& \prod_{i<j=1}^{N_{1}}\left|\vec{x}_{i}-\vec{x}_{j}\right|^{2 m} \prod_{i<j=1}^{N_{2}}\left|\vec{y}_{i}-\vec{y}_{j}\right|^{2 m} \prod_{i=1}^{N_{1}} \prod_{j=1}^{N_{2}}\left|\vec{x}_{i}-\vec{y}_{j}\right|^{2 m} \\
& \times \exp \left\{-\frac{B}{2}\left(\sum_{i=1}^{N_{1}}\left|\vec{x}_{i}\right|^{2}+\sum_{i=1}^{N_{2}}\left|\vec{y}_{i}\right|^{2}\right)\right\} \\
& \times \exp \left\{-\frac{m v_{s}}{\omega_{c}}\left(\sum_{i, j=1}^{N_{1}} \frac{1}{\left|\vec{x}_{i}-\vec{x}_{j}\right|}+\sum_{i, j=1}^{N_{2}} \frac{1}{\left|\vec{y}_{i}-\vec{y}_{j}\right|}-2 \sum_{i=1}^{N_{1}} \sum_{j=1}^{N_{2}} \frac{1}{\left|\vec{x}_{i}-\vec{y}_{j}\right|}\right)\right\}
\end{aligned}
$$

where the coordinates $\vec{x}_{i}$ are in plane $1, \vec{y}_{i}$ are in plane 2 , and $N_{1}=N_{2}=\frac{N}{2}$ for this state. 
Notice that this ground state wave function is not exactly the same as the $(m, m, m)$ Halperin wave function, to which the true ground state approaches as $d \rightarrow 0$. There is an extra factor which comes from the fact that there is a gapless mode in the spectrum of collective excitations. This contribution is analogous to the phonon contribution to the wave function of superfluid $\mathrm{He}_{4}$, and just as in that problem, it is essential to obtain the correct properties for the spatial correlations of the ground state. This contribution is very small at long distances compared to the cyclotron radius, and it can not be written only in terms of coordinates in the lowest Landau level.

\section{Conclusions}

In this Chapter, we have reviewed the fermionic Chern-Simons theory of the 2DEG in a strong magnetic field in the regime of the fractional quantum Hall effect. We showed that, for various rational values of the filling fraction $\nu$, the mean field approximation of this theory describes a set of composite fermions filling up an integer number of effective Landau levels, in agreement with Jain's picture. This resulting ground state is non degenerate and supports a gap to all of its excitations, a property that shares with the true ground state of the system. By using standard techniques of perturbation theory one can go beyond the mean field approximation in a systematic fashion.

The theory that we reviewed here is based on a second-quantized fermion path integral approach. We showed that the problem of interacting electrons moving on a plane in the presence of an external magnetic field is equivalent to a family of systems of fermions bound to an even number of fluxes, and that this theory has the fermions coupled to a Chern-Simons gauge field with ChernSimons coupling constant $\theta=(1 / 2 \pi \times 2 s)$. The semiclassical approximation of this system has solutions which describe incompressible liquid states, Wigner crystals and soliton-like defects. We worked around the liquid-like solution. This mean field solution, or Average Field Approximation (AFA), is seen to violate Galilean invariance explicitly. At this level of the approximation, the center of mass of the system executes a cyclotron-like motion at the effective cyclotron frequency. In this sense the gaussian (or semiclassical) fluctuations are essential to restore the original symmetries of the problem. We saw that, order-by-order in the semiclassical expansion, the response functions obey the correct symmetry properties required by Galilean and Gauge invariance, and by the incompressibility of the fluid. We showed that, already at the semiclassical or gaussian level, the low-momentum limit of the density correlation function saturates the $f$-sum rule, and in that sense this result is exact, $i$. e., it can not be renormalized by higher order corrections. We showed how our methods can be 
used to calculate the density-current correlation functions and, from them, to extract the Hall conductance. We found that it has the correct value already at the gaussian level of the approximation. We also obtained the spectrum of collective excitations in the low-momentum limit for short-range and for Coulomb interparticle pair potentials.

We have also presented a generalization of our approach to the study of the FQHE in double-layer systems and for unpolarized and partially polarized single-layer systems. These systems have a richer class of behaviors and excitation spectra. We have used the fermionic Chern-Simons theory to investigate the physics of these states, including results on their response functions and for the quantum numbers of their topological excitations. We have also given a specific realization of Haldane's criterion for topological stability.

Finally, we gave an explicit construction of the ground state wave functions from our semiclassical field theory. We show that their universal properties are a consequence of general principles, i. e., incompressibility and Galilean invariance which determine the analytic structure of the equal-time density correlation functions at long distances.

\section{Acknowledgments}

EF wishes to thank the Theoretical Physics Group of the Department of Physics of Oxford University, where part of this work was written, for its kind hospitality. This work was supported in part by the National Science Foundation through the grant NSF DMR94-24511 at the University of Illinois at Urbana-Champaign, and by a Glasstone Research Fellowship in Sciences and a Wolfson Junior Research Fellowship (AL).

\section{References}

1. A. Lopez and E. Fradkin, Phys. Rev. B 44, 5246 (1991).

2. J.Jain, Phys. Rev. Lett. 63, 199 (1989).

3. J.Jain, Phys. Rev. B 40, 8079 (1989).

4. F.Wilczek, Phys. Rev. Lett. 48, 1144 (1982).

5. S.Deser, R.Jackiw and S.Templeton, Phys. Rev. Lett. 48, 372 (1982).

6. J.Schonfeld, Nucl. Phys. B 185, 157 (1981).

7. E. Witten, Comm. Math. Phys. 121, 351 (1989).

8. F. Wilczek, Phys. Rev. Lett. 49, 957 (1982).

9. J. M. Leinaas and J. Myrheim, Nuovo Cimento 37B, 132 (1977).

10. A.M.Polyakov, Mod. Phys. Lett. A 3, 325 (1988).

11. E.Fradkin, Phys. Rev. Lett. 63, 322 (1989).

12. S. Kivelson, D.H. Lee and S.C. Zhang, Phys. Rev. B 46, 2223 (1992). 
13. S.Coleman, Aspects of Symmetry, (Cambridge University Press, Cambridge, 1985).

14. R.Rajaraman, Solitons and Instantons, (North-Holland, Amsterdam, 1987).

15. D. Bohm and D. Pines, Phys. Rev. 92, 609 (1953); Phys. Rev. 92, 626 (1953).

16. B. I. Halperin, P. A. Lee and N. Read, Phys. Rev. B 47, 7312 (1993).

17. R.B.Laughlin in The Quantum Hall Effect, R.Prange and S.Girvin editors (Springer-Verlag, New York, 1989).

18. S.Randjbar-Daemi, A.Salam and J.Strathdee, Nucl. Phys. B 340, 403 (1990).

19. Y.H.Chen, F.Wilczek, E.Witten and B.I.Halperin, Int. Jour. Mod. Phys. B 3, 1001 (1989).

20. Walter Kohn, Phys. Rev. 123, 1242 (1961).

21. P. Ramond, Field Theory; A Modern Primer, (Addison-Wesley, Redwood City ,1989).

22. A. Lopez and E. Fradkin, Phys. Rev. B 47, 7080 (1993).

23. S.H. Simon and B.I. Halperin, Phys. Rev. B 48, 17365 (1993).

24. C. Kallin and B.I. Halperin, Phys. Rev. B 30, 5655 (1984).

25. S. Girvin, A. MacDonald and P. Platzman, Phys. Rev. Lett. 54, 581 (1985); Phys. Rev. B 33, 2481 (1986).

26. S.C. Zhang, Int. Jour. Mod. Phys. B 6, 25 (1992).

27. M.V. Klein in Light Scattering in Solids I, M. Cardona, editor ( SpringerVerlag, New York, 1983).

28. Q. Nui, D.J. Thouless and Y.S. Wu, Phys. Rev. B 31, 3372 (1985).

29. M. Kohmoto, Ann. Phys. 160, 343 (1985).

30. E. Fradkin, Field Theories of Condensed Matter Systems (AddisonWesley, Redwood City, 1991).

31. D. Arovas, J.R. Schrieffer and F. Wilczek, Phys. Rev. Lett. 53, 722 (1984).

32. R.L. Willet, J.P. Eisenstein, H.L. Stormer, D.C. Tsui, A.C. Gossard, and J.H. English, Phys. Rev. Lett. 59, 1776 (1987).

33. F.D.M. Haldane and E.H. Rezayi, Phys. Rev. Lett. 60, 956 (1988).

34. A.H. MacDonald, P.M. Platzman, and G.S. Boebinger, Phys. Rev. Lett. 65, 775 (1990); L. Brey, Phys. Rev. Lett. 65, 903 (1990).

35. G.S. Boebinger, H.W. Jiang, L.N. Pfeiffer, and K.W. West, Phys. Rev. Lett. 64, 1793 (1990); Y.W. Suen, J. Jo, M.B. Santos, L.W. Engel, S.W. Hwang, and M. Shayegan, Phys. Rev. B 44, 5947 (1991).

36. Y.W. Suen, L.W. Engel, M.B. Santos, M. Shayegan, and D.C. Tsui, Phys. Rev. Lett. 68, 1379 (1992); J.P. Eisenstein, G.S. Boebinger, L.N. 
Pfeiffer, K.W. West, and Song He, Phys. Rev. Lett. 68, 1383 (1992).

37. A. Lopez and E. Fradkin, Phys. Rev. B 51, 4347 (1995).

38. S.L. Sondhi, A. Karlhede, S.A. Kivelson, and E. Rezayi, Phys. Rev. B 47, 16419 (1993).

39. X. G. Wen and Q. Niu, Phys. Rev. B 41, 9377 (1990), X. G. Wen, Adv. in Phys. 44, 405 (1995).

40. F.D.M. Haldane, Phys. Rev. Lett. 74, 2090 (1995).

41. X.G. Wen and A. Zee, Phys. Rev. Lett. 69, 1811 (1992); ibid., Phys. Rev. B 47, 2265 (1993).

42. B.I. Halperin, Helv. Phys. Acta 56, 75 (1983).

43. F. Wilczek, Phys. Rev. Lett. 69, 132 (1992).

44. Z.F. Ezawa and A. Iwazaki, Int. Jour. Mod. Phys. B 6, 25 (1992); Phys. Rev. B 47, 7295 (1993); Phys. Rev. B 48, 15189 (1993).

45. A.R. Hamilton, M.Y. Simmons, F.M. Bolton, N.K. Patel, I.S. Millard, J.T. Nicholls, D. A. Ritchie, and M. Pepper, Phys. Rev. B 54, R5259 (1996).

46. X.G. Wen and A. Zee, Phys. Rev. B 46, 2290 (1992).

47. K. Yang, K. Moon, L. Zheng, A.H. MacDonald, S. M. Girvin, D. Yoshioka and S. C. Zhang, Phys. Rev. Lett. 72, 732 (1994).

48. A. Balatsky and E. Fradkin, Phys. Rev. B 43, 10622 (1992).

49. J. Frohlich, T. Kerler, and P. A. Marchetti, Nucl. Phys. B 374, 111 (1992).

50. L. Belkhir and J.K. Jain, Phys. Rev. Lett. 70, 643 (1993).

51. D.H. Lee and C. Kane, Phys. Rev. Lett. 64, 1313 (1990).

52. N. Read and G. Moore, Nucl. Phys. B 360, 362 (1991).

53. V. Kalmeyer and R.B. Laughlin, Phys. Rev. Lett. 59, 2095 (1988).

54. A. Lopez and E. Fradkin ,Phys. Rev. Lett. 69, 2126 (1992).

55. E. Fradkin, Nucl. Phys. B 389, 587 (1993).

56. Song He, S. Das Sarma and X.C. Xie, Phys. Rev. B 47, 4394 (1993). 